\title{
The effects of fixed and removable orthodontic retainers: a systematic review
}

\author{
Dalya Al-Moghrabi ${ }^{1^{*}}$ (D), Nikolaos Pandis ${ }^{2}$ and Padhraig S Fleming ${ }^{1}$
}

\begin{abstract}
Objective: In the view of the widespread acceptance of indefinite retention, it is important to determine the effects of fixed and removable orthodontic retainers on periodontal health, survival and failure rates of retainers, cost-effectiveness, and impact of orthodontic retainers on patient-reported outcomes.

Methods: A comprehensive literature search was undertaken based on a defined electronic and gray literature search strategy (PROSPERO: CRD42015029169). The following databases were searched (up to October 2015); MEDLINE via OVID, PubMed, the Cochrane Central Register of Controlled Trials, LILACS, BBO, ClinicalTrials.gov, the National Research Register, and ProQuest Dissertation and Thesis database. Randomized and non-randomized controlled clinical trials, prospective cohort studies, and case series (minimum sample size of 20) with minimum follow-up periods of 6 months reporting periodontal health, survival and failure rates of retainers, cost-effectiveness, and impact of orthodontic retainers on patient-reported outcomes were identified. The Cochrane Collaboration's Risk of Bias tool and Newcastle-Ottawa Scale were used to assess the quality of included trials.

Results: Twenty-four studies were identified, 18 randomized controlled trials and 6 prospective cohort studies. Of these, only 16 were deemed to be of high quality. Meta-analysis was unfeasible due to considerable clinical heterogeneity and variations in outcome measures. The mean failure risk for mandibular stainless steel fixed retainers bonded from canine to canine was 0.29 (95\% confidence interval $[\mathrm{Cl}] 0.26,0.33$ ) and for those bonded to canines only was 0.25 (95\% Cl: 0.16, 0.33). A meta-regression suggested that failure of fixed stainless steel mandibular retainers was not directly related to the period elapsed since placement $(P=0.938)$.
\end{abstract}

Conclusion: Further well-designed prospective studies are needed to elucidate the benefits and potential harms associated with orthodontic retainers.

Keywords: Orthodontic retainer, Periodontal, Survival rate, Failure rate, Cost-effectiveness, Patient-reported outcomes

\section{Review}

Introduction

Retention procedures are considered necessary to maintain the corrected position of teeth following orthodontic treatment and to mitigate against characteristic agerelated changes which, if unchecked, are known to culminate in mandibular anterior crowding [1]. Retention procedures are continually being refined with a recognition that existing protocols are infallible [2]. Nevertheless, both fixed and removable retainers continue to be in vogue, although adjunctive procedures including

\footnotetext{
* Correspondence: d.almoghrabi@gmul.ac.uk

'Barts and The London School of Medicine and Dentistry, Queen Mary

University of London, London E1 2AD, UK

Full list of author information is available at the end of the article
}

interproximal enamel reduction and minor oral surgical procedures have also been advocated.

A recent Cochrane review exposed a lack of highquality evidence to favor one method of retention over another in terms of stability [3]. Given this absence of definitive evidence, retainer selection is often based on individual preference. This is evidenced by marked geographical variation with maxillary Hawley or vacuumformed retainers and mandibular fixed lingual retainers with full-time wear of removable retainers most popular in the USA [4, 5]. In Australia and New Zealand, mandibular fixed and maxillary vacuum-formed retainers are shown to be the most prevalent combination [6], while a preference for the use of fixed retainers in both arches has been shown in the Netherlands [7]. 
The duration of wear of orthodontic retainers has long been a dilemma in orthodontics. However, there is now widespread acceptance of the necessity for indefinite retention to minimize both relapse and maturational changes [5, 8]. Prolonged retention may pose increased risk to the periodontium and dental hard tissues; it is therefore important to investigate the implications of the long-term use of fixed and removable retainers on the supporting tissues $[3,9,10]$.

A further consideration is patient experiences of retention and compliance with prolonged retention regimes; it is intuitive to expect that co-operation with retention regimes would decline over time. Moreover, both fixed and removable retainers are prone to breakage, loss, and degradation $[2,11]$. Repeated breakage and requirement for replacement may have implications for the costeffectiveness of both fixed and removable approaches. There is however limited evidence concerning the costeffectiveness of either approach $[12,13]$.

The primary aim of this systematic review was to determine the influence of fixed and removable orthodontic retainers on periodontal health in patients who have completed orthodontic treatment with fixed appliances. A secondary aim was to evaluate survival and failure rates, impact of orthodontic retainers on patient-reported outcomes, and cost-effectiveness.

\section{Materials and methods}

This protocol for this systematic review was registered on PROSPERO (www.crd.york.ac.uk/prospero; CRD42015 029169). The following selection criteria were applied:

- Study design: randomized and non-randomized controlled clinical trials, prospective cohort studies, and case series (with a minimum sample size of 20 patients) with minimum follow-up periods of 6 months

- Participants: patients having had orthodontic treatment with fixed or removable appliances followed by orthodontic retention

- Interventions: fixed retainers, removable retainers, and interproximal reduction

- Outcome measures: periodontal outcomes, survival and failure rates (including detachment of fixed retainers, breakages, retainer loss, or the need for replacement), patient-reported outcomes, and cost-effectiveness measures

\section{Search strategy for identification of studies}

The following databases were searched up to October 2015 without language restrictions: MEDLINE via OVID (Appendix 1), PubMed, the Cochrane Central Register of Controlled Trials (CENTRAL), and LILACS and $\mathrm{BBO}$ databases. Unpublished trials were searched electronically using ClinicalTrials.gov (www.clinicaltrials.gov), the National Research Register (www.controlled-trials.com), and ProQuest Dissertation and Thesis database (http://pqdtopen.proquest.com).

\section{Assessment of relevance, validity, and data extraction}

Full texts of relevant abstracts were retrieved. Data was tabulated using pre-piloted data collection forms by two authors (DA, PSF). Data extracted included: (1) study design; (2) sample: size, demographics, and clinical characteristics; (3) intervention: fixed appliances, removable appliances, or interproximal reduction; (4) follow-up period; (5) maxillary/mandibular arch; and (6) outcomes (primary and secondary).

\section{Risk of bias (quality) assessment}

For randomized controlled trials sequence generation, allocation concealment, blinding of outcome assessors, incomplete outcome data, selective reporting, and other biases were assessed using the Cochrane Collaboration's Risk of Bias tool. Any disagreement was resolved by joint discussion (DA, PSF). Only studies at low or unclear risk of bias overall were to be included in the meta-analysis. The methodological quality of the included nonrandomized studies was assessed using the NewcastleOttawa Scale. Studies adjudged to be of moderate or high methodological quality overall (more than five stars) were to be included in the meta-analysis. The authors of the included studies were contacted for clarification if required.

\section{Strategy for data synthesis}

Clinical heterogeneity was assessed according to the treatment interventions, wear regimen for removable retainers, measurement approach, and location of the retainers. For periodontal outcomes, the index used and surfaces examined were considered. Statistical heterogeneity was to be assessed by inspecting a graphic display of the estimated treatment effects from individual trials with associated $95 \%$ confidence intervals. Heterogeneity would be quantified using $I$-squared with values above $50 \%$ indicative of moderate to high heterogeneity which might preclude meta-analysis. A weighted treatment effect was to be calculated, and the results for retainer failure were expressed as odds ratios. All statistical analyses were undertaken using the Stata statistical software package (version 12.1; StataCorp, College Station, Tex).

\section{Results}

\section{Description of the included studies}

Sixty-four were considered potentially relevant to the review. Following retrieval of the full-text articles, 36 studies were excluded. Overall, 24 studies met the inclusion 
criteria (Fig. 1). Reasons for exclusion at the final stage are presented (Appendix 2). The study design, characteristics of participants, comparison groups, follow-up period, and the outcomes of the included studies are presented in Table 1.
Risk of bias/methodological quality of included studies

The random sequence generation was adequately performed in 12 studies [11-22]. The assessor was adequately blinded in six trials $[13,14,16,19,20,22]$. Overall, 11 randomized clinical trials were judged to be

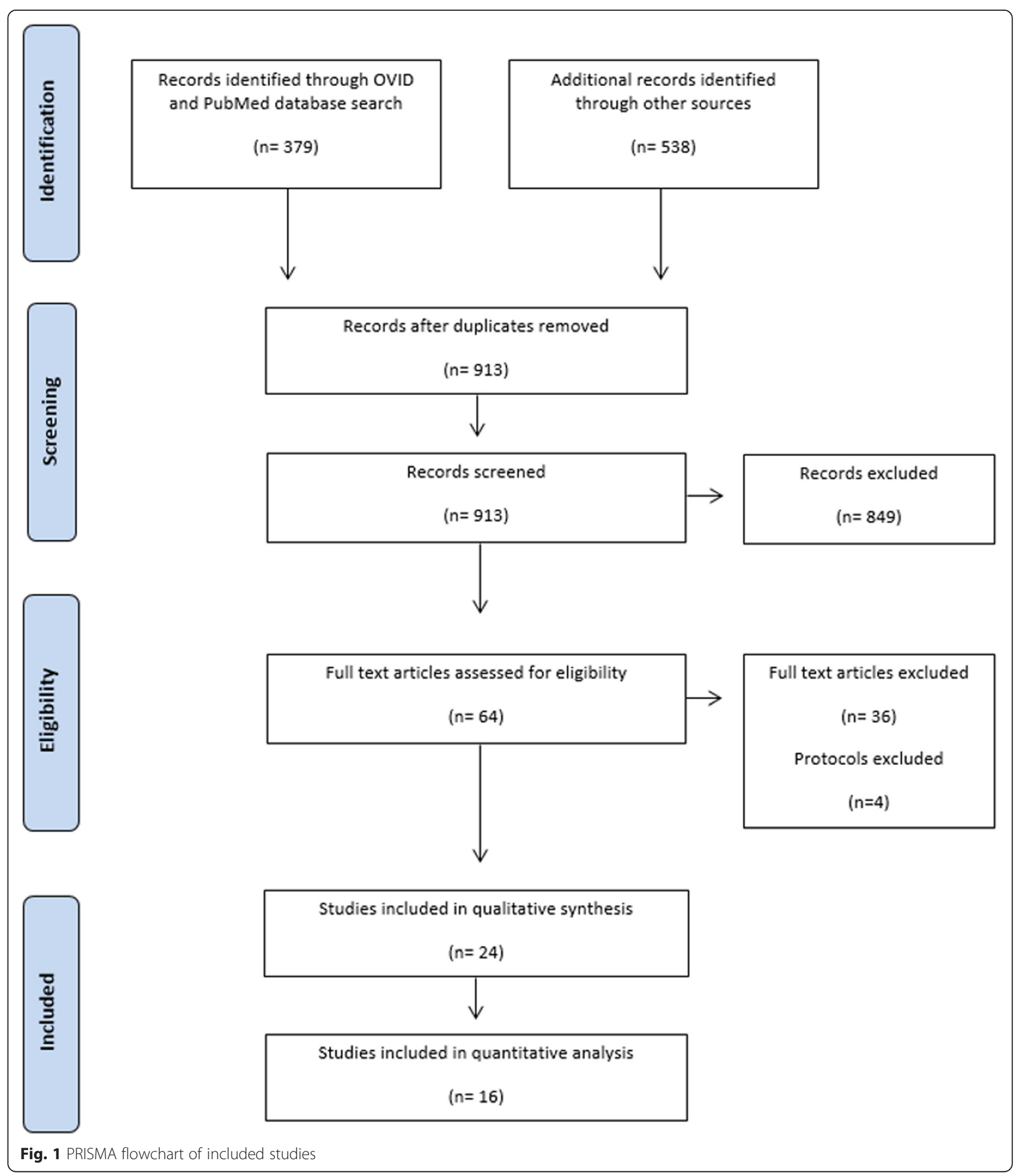


Table 1 Characteristics of included trials ( $n=24)$

\begin{tabular}{|c|c|c|c|c|c|c|c|}
\hline Study & Design & Participants (overall) & Intervention/comparison & Wear (part-time/full-time) & $\begin{array}{l}\text { Follow-up } \\
\text { period } \\
(\text { mean } \pm \text { SD) }\end{array}$ & Dental arch & Outcomes \\
\hline $\begin{array}{l}\text { Al-Nimri et al. } \\
2009[25]\end{array}$ & $\begin{array}{l}\text { Prospective } \\
\text { cohort study }\end{array}$ & $n=62(18 \mathrm{M}, 44 \mathrm{~F})$ & $\begin{array}{l}\text { - } 0.036 " \text { round stainless steel } \\
\text { fixed retainer (canines only) } \\
(n=31 ; \text { mean age, } 20.23 \pm \\
3.8 \text { years) } \\
-0.015^{\prime \prime} \text { multistrand fixed } \\
\text { retainer ( } n=31 \text {; mean age, } \\
19.97 \pm 4.2 \text { years) }\end{array}$ & & $\begin{array}{l}21.31 \text { months } \\
19.35 \text { months }\end{array}$ & Mandibular anterior teeth & $\begin{array}{l}\text { Plaque Index, Gingival Index, } \\
\text { retainer failure, Oral Hygiene } \\
\text { Index, Irregularity Index }\end{array}$ \\
\hline $\begin{array}{l}\text { Bazargani et al. } \\
2012 \text { [14] }\end{array}$ & RCT & $\begin{array}{l}n=51 \\
\text { Overall mean age, } \\
18.3 \pm 1.3 \text { years }\end{array}$ & $\begin{array}{l}-0.0195 " \text { multistrand fixed } \\
\text { retainer with two-step bonded } \\
\text { resin adhesive }(n=25) \\
-0.0195^{\prime \prime} \text { multistrand fixed } \\
\text { retainer with non-resin adhesive } \\
(n=26)\end{array}$ & & $\begin{array}{l}24.4 \pm 4.7 \\
\text { months }\end{array}$ & Mandibular anterior teeth & $\begin{array}{l}\text { Retainer failure, calculus } \\
\text { accumulation, discoloration } \\
\text { around composite pads }\end{array}$ \\
\hline $\begin{array}{l}\text { Störmann and } \\
\text { Ehmer } 2002 \text { [15] }\end{array}$ & RCT & $\begin{array}{l}n=98 \\
\text { Overall age range, } \\
13-17 \text { years }\end{array}$ & $\begin{array}{l}-0.0195^{\prime \prime} \text { Respond } d^{\oplus} \text { fixed retainer } \\
(n=30) \\
-0.0215^{\prime \prime} \text { Respond } d^{\oplus} \text { fixed retainer } \\
(n=36) \\
\text { - Prefabricated fixed retainer } \\
\text { (canines only) }(n=32)\end{array}$ & & 24 months & Mandibular anterior teeth & $\begin{array}{l}\text { Bleeding on probing, Plaque } \\
\text { Index, failure rate, aesthetic } \\
\text { problems, patient discomfort, } \\
\text { Little's irregularity index, } \\
\text { occlusal discrepancies, } \\
\text { intercanine width }\end{array}$ \\
\hline \multirow[t]{3}{*}{$\begin{array}{l}\text { Tynelius et al. } \\
2014 \text { [13] }\end{array}$} & RCT & $\begin{array}{l}n=75(30 \mathrm{M}, 45 \mathrm{~F}) \\
\text { Overall mean age, } \\
14.3 \pm 1.5 \text { years }\end{array}$ & $\begin{array}{l}\text { - Vacuum-formed retainer in the } \\
\text { maxilla and 0.7-mm spring hard } \\
\text { wire fixed retainer in the } \\
\text { mandibule (canines only) }(n=25 \text { ) }\end{array}$ & $\begin{array}{l}\text { Full-time for } 2 \text { days } \\
\text { followed by part-time } \\
\text { for } 1 \text { year. Every other } \\
\text { night in the second year }\end{array}$ & 24 months & $\begin{array}{l}\text { Maxillary and mandibular } \\
\text { dentition }\end{array}$ & $\begin{array}{l}\text { Cost-effectiveness and } \\
\text { societal costs }\end{array}$ \\
\hline & & & $\begin{array}{l}\text { - Vacuum-formed retainer in the } \\
\text { maxilla and interproximal enamel } \\
\text { reduction in the mandibular } \\
\text { anterior teeth }(n=25)\end{array}$ & $\begin{array}{l}\text { Full-time for } 2 \text { days } \\
\text { followed by part-time } \\
\text { for } 1 \text { year. Every other } \\
\text { night in the second year }\end{array}$ & & & \\
\hline & & & - Prefabricated positioner $(n=25)$ & $\begin{array}{l}\text { Part-time for } 1 \text { year, } \\
\text { followed by every other } \\
\text { night in the second year }\end{array}$ & & & \\
\hline $\begin{array}{l}\text { Torkan et al. } \\
2014 \text { [16] }\end{array}$ & RCT & $n=30(10 \mathrm{M}, 20 \mathrm{~F})$ & $\begin{array}{l}\text { - Fiber-reinforced resin composite } \\
\text { fixed retainer ( } n=15 \text {; mean age, } \\
16.2 \pm 1.9) \\
\text { - } 0.0175 \text { " Multistrand stainless } \\
\text { steel fixed retainer }(n=15 ; \text { mean } \\
\text { age, } 15.7 \pm 2.1 \text { years) }\end{array}$ & & 6 months & $\begin{array}{l}\text { Maxillary and mandibular } \\
\text { anterior teeth }\end{array}$ & $\begin{array}{l}\text { Plaque Index, Calculus Index, } \\
\text { Gingival Index, bleeding on } \\
\text { probing, width of periodontal } \\
\text { ligament }\end{array}$ \\
\hline $\begin{array}{l}\text { Sfondrini et al. } \\
2014 \text { [17] }\end{array}$ & RCT & $\begin{array}{l}n=87 \text { ( } 35 \mathrm{M}, 52 \mathrm{~F}) \\
\text { Overall average age, } \\
24 \text { years (14-62 years) }\end{array}$ & $\begin{array}{l}\text { - } 0.5 \text {-mm silanized-treated glass } \\
\text { fiber-reinforced composite resin } \\
\text { fixed retainer }(n=40) \\
\text { - } 0.0175^{\prime \prime} \text { multistrand stainless } \\
\text { steel fixed retainer }(n=47)\end{array}$ & & 12 months & Mandibular anterior teeth & Bond adhesive failure \\
\hline
\end{tabular}


Table 1 Characteristics of included trials ( $n=24)$ (Continued)

\begin{tabular}{|c|c|c|c|c|c|c|c|}
\hline \multirow[t]{2}{*}{$\begin{array}{l}\text { Ardeshna et al. } \\
2011 \text { [26] }\end{array}$} & \multirow[t]{2}{*}{$\begin{array}{l}\text { Prospective } \\
\text { cohort study }\end{array}$} & \multirow[t]{2}{*}{$\begin{array}{l}n=56 \text { (76 fixed } \\
\text { retainers) }\end{array}$} & \multicolumn{2}{|l|}{$\begin{array}{l}\text { - 0.53- or } 1.02-\mathrm{mm} \text { fiber-reinforced } \\
\text { thermoplastic fixed retainer with } \\
\text { polyethylene terephthalate glycol } \\
\text { matrix resin }\end{array}$} & 24 months & $\begin{array}{l}\text { Maxillary anterior teeth } \\
\text { ( } 2 \text { retainers), mandibular } \\
\text { anterior teeth ( } 21 \text { retainers, } \\
6 \text { of them were bonded } \\
\text { to canines only) }\end{array}$ & \multirow[t]{2}{*}{ Survival and failure rates } \\
\hline & & & $\begin{array}{l}\text { - } 0.53-\text { or } 1.02-\mathrm{mm} \text { fiber-reinforced } \\
\text { thermoplastic fixed retainer with } \\
\text { polycarbonate matrix resin }\end{array}$ & & & $\begin{array}{l}\text { Maxillary anterior teeth } \\
\text { (14 retainers), mandibular } \\
\text { anterior teeth ( } 39 \\
\text { retainers, } 5 \text { of them } \\
\text { were canines only) }\end{array}$ & \\
\hline $\begin{array}{l}\text { Salehi et al. } \\
2013 \text { [18] }\end{array}$ & $\mathrm{RCT}$ & $\begin{array}{l}n=142(59 \mathrm{M}, 83 \mathrm{~F}) \\
\text { Overall age range, } \\
14-28 \text { years }\end{array}$ & $\begin{array}{l}\text { - Polyethylene woven ribbon fixed } \\
\text { retainer ( } n=68 \text {; mean age, } 18.1 \pm \\
5.23 \text { years) } \\
-0.0175^{\prime \prime} \text { multistrand stainless steel } \\
\text { fixed retainer ( } n=74 \text {; mean age, } \\
18.2 \pm 4.81 \text { years) }\end{array}$ & & 18 months & $\begin{array}{l}\text { Maxillary and mandibular } \\
\text { anterior teeth }\end{array}$ & Survival and failure rates \\
\hline \multirow[t]{2}{*}{$\begin{array}{l}\text { Hichens et al. } \\
2007 \text { [12] }\end{array}$} & \multirow[t]{2}{*}{$\mathrm{RCT}$} & \multirow{2}{*}{$\begin{array}{l}n=355(350 \\
\text { questionnaires } \\
\text { completed at } 6 \text { months }) \\
(155 \mathrm{M}, 242 \mathrm{~F})^{\mathrm{a}} \\
\text { Overall mean age }= \\
14-15 \text { years }\end{array}$} & - Hawley retainer ( $n=172)$ & $\begin{array}{l}\text { Full-time for } 3 \text { months } \\
\text { followed by part-time for } \\
3 \text { months }\end{array}$ & \multirow[t]{2}{*}{6 months } & \multirow[t]{2}{*}{$\begin{array}{l}\text { Maxillary and mandibular } \\
\text { dentition }\end{array}$} & \multirow[t]{2}{*}{$\begin{array}{l}\text { Cost-effectiveness, patient } \\
\text { satisfaction, failure rate, } \\
\text { Little's irregularity index }\end{array}$} \\
\hline & & & - Vacuum-formed retainer $(n=183)$ & $\begin{array}{l}\text { Full-time for } 1 \text { week, } \\
\text { followed by part-time }\end{array}$ & & & \\
\hline $\begin{array}{l}\text { Bolla et al. } \\
2012 \text { [39] }\end{array}$ & $\mathrm{RCT}$ & $n=85(29 M, 56 F)$ & $\begin{array}{l}\text { - Glass fiber-reinforced fixed retainer } \\
\text { ( } n=40 ; \text { mean age for M, } 23.4 \text { years; } \\
\text { mean age for } F, 20.2 \text { years) } \\
\text { - } 0.0175^{\prime \prime} \text { multistrand stainless steel } \\
\text { fixed retainer ( } n=45 \text {; mean age } \\
\text { for } M, 24.1 \text { years; mean age for } F \text {, } \\
22.6 \text { years) }\end{array}$ & & 6 years & $\begin{array}{l}\text { Maxillary } 2-2 \text { ( } 14 \text { retainers) } \\
\text { and mandibular ( } 34 \\
\text { retainers) anterior teeth } \\
\text { Maxillary } 2-2 \text { (18 retainers) } \\
\text { and mandibular ( } 32 \\
\text { retainers) anterior teeth }\end{array}$ & $\begin{array}{l}\text { Bond failure and breakage } \\
\text { of retainers }\end{array}$ \\
\hline $\begin{array}{l}\text { Tacken et al. } \\
2010 \text { [31] }\end{array}$ & $\mathrm{RCT}$ & $\begin{array}{l}n=274(135 \mathrm{M}, 139 \mathrm{~F})^{\mathrm{a}} \\
\text { Overall mean age, } \\
14 \text { years }\end{array}$ & $\begin{array}{l}\text { - Glass fiber-reinforced fixed retainer } \\
\text { ( } 500 \text { unidirectional glass fibers) } \\
\text { ( } n=45 ; \text { mean age, } 14.8 \text { years } \pm \\
1.3 \text { years) } \\
\text { - Glass fiber-reinforced fixed } \\
\text { retainer ( } 1000 \text { unidirectional glass } \\
\text { fibers) ( } n=48 ; \text { mean age, } 14.6 \\
\text { years } \pm 2.7 \text { years) } \\
\text { - } 0.0215^{\prime \prime} \text { coaxial fixed retainer } \\
\text { ( } n=91 ; \text { mean age, } 15 \text { years } \pm \\
1.3 \text { years) } \\
\text { - Untreated control }(n=90)\end{array}$ & & 24 months & $\begin{array}{l}\text { Maxillary } 2-2 \text { and } \\
\text { mandibular anterior } \\
\text { teeth }\end{array}$ & $\begin{array}{l}\text { Failure rate, modified } \\
\text { gingival index (MGl), } \\
\text { bleeding on probing, } \\
\text { Plaque Index (PI) }\end{array}$ \\
\hline $\begin{array}{l}\text { Bovali et al. } \\
2014 \text { [19] }\end{array}$ & $\mathrm{RCT}$ & $\begin{array}{l}n=63(28 \mathrm{M}, 35 \mathrm{~F}) \\
\text { Overall age range: } \\
12-38 \text { years }\end{array}$ & $\begin{array}{l}\text { - Direct bonding of } 0.0215 " \\
\text { multistrand stainless steel fixed } \\
\text { retainer }(n=31 \text {; mean age, }\end{array}$ & & 6 months & Mandibular anterior teeth & $\begin{array}{l}\text { Failure rate, time to fit } \\
\text { retainers }\end{array}$ \\
\hline
\end{tabular}


Table 1 Characteristics of included trials ( $n=24)$ (Continued)

\begin{tabular}{|c|c|c|c|c|c|c|c|}
\hline & & & $\begin{array}{l}\text { - Indirect bonding of } 0.0215^{\prime \prime} \\
\text { multistrand stainless steel fixed } \\
\text { retainer ( } n=32 ; \text { mean age, } \\
17.2 \pm 3.1 \text { years) }\end{array}$ & & & & \\
\hline $\begin{array}{l}\text { Pandis et al. } \\
2013 \text { [20] }\end{array}$ & $\mathrm{RCT}$ & $\begin{array}{l}n=220(60 \mathrm{M}, 160 \mathrm{~F}) \\
\text { Overall median age, } \\
16(\mathrm{IQR} 2) \text { years } \\
\text { Overall age range, } \\
12-47 \text { years }\end{array}$ & $\begin{array}{l}\text { - } 0.022^{\prime \prime} \text { multistrand stainless steel } \\
\text { fixed retainer bonded with } \\
\text { chemical-cured composite }(n=110 ; \\
\text { median age, } 16 \text { (IQR 2) years) } \\
\text { - } 0.022^{\prime \prime} \text { multistrand stainless } \\
\text { steel fixed retainer bonded with } \\
\text { light-cured composite ( } n=110 ; \\
\text { median age, } 16 \text { (IQR 2) years) }\end{array}$ & & $\begin{array}{l}\text { Median follow- } \\
\text { up period: } \\
2.19 \text { years } \\
\text { Range, } 0.003- \\
3.64 \text { years }\end{array}$ & Mandibular anterior teeth & $\begin{array}{l}\text { Failure rate, adhesive } \\
\text { remnant index scores }\end{array}$ \\
\hline \multirow{2}{*}{$\begin{array}{l}\text { Sun et al. } \\
2011[11]\end{array}$} & \multirow[t]{2}{*}{$\mathrm{RCT}$} & \multirow{2}{*}{$\begin{array}{l}n=111 \\
\text { Overall mean age, } \\
14.7 \text { years } \\
\text { Overall age range, } \\
12-17 \text { years }\end{array}$} & - Hawley retainer $(n=56)$ & Full-time & \multirow[t]{2}{*}{12 months } & \multirow{2}{*}{$\begin{array}{l}\text { Maxillary and mandibular } \\
\text { dentition }\end{array}$} & \multirow[t]{2}{*}{ Survival and failure rates } \\
\hline & & & - Vacuum-formed retainer $(n=55)$ & Full-time & & & \\
\hline \multirow{2}{*}{$\begin{array}{l}\text { Xu et al. } \\
2011[40]\end{array}$} & \multirow[t]{2}{*}{ RCT } & \multirow{2}{*}{$\begin{array}{l}n=40(16 \mathrm{M}, 29 \mathrm{~F}) \\
\text { Overall mean age, } \\
13-16 \text { years }\end{array}$} & - Vacuum-formed retainer $(n=25)$ & Full-time & \multirow[t]{2}{*}{12 months } & \multirow{2}{*}{$\begin{array}{l}\text { Maxillary and mandibular } \\
\text { dentition }\end{array}$} & \multirow{2}{*}{$\begin{array}{l}\text { Overjet, overbite, } \\
\text { intercanine width, } \\
\text { intermolar width, Little's } \\
\text { irregularity index, Calculus } \\
\text { Index scores, failure rate }\end{array}$} \\
\hline & & & $\begin{array}{l}-0.0195 " \text { multistrand stainless steel } \\
\text { fixed retainer with Hawley retainer } \\
(n=15)\end{array}$ & Part-time & & & \\
\hline $\begin{array}{l}\text { Rose et al. } \\
2002 \text { [41] }\end{array}$ & $\mathrm{RCT}$ & $\begin{array}{l}n=20(12 \mathrm{M}, 8 \mathrm{~F}) \\
\text { Overall mean age, } \\
22.4 \pm 9.7 \text { years }\end{array}$ & $\begin{array}{l}\text { - } 1 \text {-mm polyethylene woven ribbon } \\
\text { fixed retainer }(n=10) \\
\text { - } 0.0175^{\prime \prime} \text { multistrand stainless steel } \\
\text { fixed retainer }(n=10)\end{array}$ & & 24 months & Mandibular anterior teeth & $\begin{array}{l}\text { Patient acceptance and } \\
\text { preference, survival of } \\
\text { retainers, amount of } \\
\text { calculus, demineralisation, } \\
\text { caries }\end{array}$ \\
\hline $\begin{array}{l}\text { Liu et al. } \\
2010[23]\end{array}$ & $\mathrm{RCT}$ & $n=60$ & $\begin{array}{l}\text { - } 0.75 \text {-mm fiber-reinforced composite } \\
\text { fixed retainer }(n=30) \\
\text { - 0.9-mm multistrand stainless steel } \\
\text { fixed retainer }(n=30)\end{array}$ & & 12 months & $\begin{array}{l}\text { Mandibular anterior } \\
\text { teeth }\end{array}$ & $\begin{array}{l}\text { Bleeding index, pocket } \\
\text { depth, failure rate }\end{array}$ \\
\hline $\begin{array}{l}\text { Taner et al. } \\
2012 \text { [27] }\end{array}$ & $\begin{array}{l}\text { Prospective } \\
\text { cohort study }\end{array}$ & $n=66(14 \mathrm{M}, 52 \mathrm{~F})$ & $\begin{array}{l}\text { - Direct bonding of } 0.016^{\prime \prime} \times 0.022^{\prime \prime} \\
\text { multistrand stainless steel dead } \\
\text { soft wire fixed retainer }(n=32 \text {; } \\
\text { mean age, } 15.96 \pm 3.21 \text { years) } \\
\text { - Indirect bonding of } 0.016^{\prime \prime} \times 0.022^{\prime \prime} \\
\text { multistrand stainless steel dead soft } \\
\text { wire fixed retainer }(n=34 \text {; mean } \\
\text { age, } 19.44 \pm 6.79 \text { years) }\end{array}$ & & 6 months & $\begin{array}{l}\text { Mandibular anterior } \\
\text { teeth }\end{array}$ & Failure rate \\
\hline \multirow[t]{3}{*}{$\begin{array}{l}\text { Artun et al. } \\
1997 \text { [28] }\end{array}$} & \multirow[t]{3}{*}{$\begin{array}{l}\text { Prospective } \\
\text { cohort study }\end{array}$} & \multirow[t]{3}{*}{$n=49$} & $\begin{array}{l}\text { - } 0.032^{\prime \prime} \text { plain fixed retainer (canines } \\
\text { only) }(n=11)\end{array}$ & & \multirow[t]{3}{*}{3 years } & \multirow[t]{3}{*}{$\begin{array}{l}\text { Mandibular anterior } \\
\text { teeth }\end{array}$} & \multirow{3}{*}{$\begin{array}{l}\text { Little's irregularity index, } \\
\text { failure rate, Plaque Index, } \\
\text { Calculus Index, Gingival } \\
\text { Index, probing attachment } \\
\text { level }\end{array}$} \\
\hline & & & $\begin{array}{l}-0.032^{\prime \prime} \text { spiral wire fixed retainer } \\
\text { (canines only) }(n=13)\end{array}$ & & & & \\
\hline & & & - 0.0205" spiral wire fixed retainer & & & & \\
\hline
\end{tabular}


Table 1 Characteristics of included trials ( $n=24)$ (Continued)

\begin{tabular}{|c|c|c|c|c|c|c|c|}
\hline $\begin{array}{l}\text { Scribante et al. } \\
2011[24]\end{array}$ & RCT & $\begin{array}{l}n=34(9 \mathrm{M}, 25 \mathrm{~F}) \\
\text { Overall mean age, } \\
14.3 \text { years }\end{array}$ & $\begin{array}{l}\text { - } 0.0175^{\prime \prime} \text { multistrand stainless steel } \\
\text { fixed retainer } \\
\text { - Polyethylene fiber-reinforced } \\
\text { resin composite fixed retainer }\end{array}$ & & 12 months & $\begin{array}{l}\text { Mandibular anterior } \\
\text { teeth }\end{array}$ & $\begin{array}{l}\text { Failure rate, patient } \\
\text { satisfaction of the } \\
\text { aesthetic result }\end{array}$ \\
\hline \multirow[t]{2}{*}{$\begin{array}{l}\text { Zachrisson, } \\
1977 \text { [29] }\end{array}$} & \multirow[t]{2}{*}{$\begin{array}{l}\text { Prospective } \\
\text { cohort study }\end{array}$} & \multirow[t]{2}{*}{$n=43$ (14-17 years) } & $\begin{array}{l}-0.032 " \text { or } 0.036 " \text { blue Elgiloy } \\
\text { fixed retainer bonded using a } \\
\text { holding wire (canines only) } \\
(n=22)\end{array}$ & & \multirow[t]{2}{*}{$\begin{array}{l}\text { Mean, } 15.7 \\
\text { months; } \\
\text { Range, } \\
\text { 12-30 months }\end{array}$} & \multirow[t]{2}{*}{$\begin{array}{l}\text { Mandibular anterior } \\
\text { teeth }\end{array}$} & \multirow[t]{2}{*}{$\begin{array}{l}\text { Failure rate, calculus } \\
\text { accumulation }\end{array}$} \\
\hline & & & $\begin{array}{l}-0.032^{\prime \prime} \text { or } 0.036 " \text { blue Elgiloy } \\
\text { fixed retainer bonded using a } \\
\text { steel ligature (canines only) } \\
(n=21)\end{array}$ & & & & \\
\hline \multirow[t]{2}{*}{$\begin{array}{l}\text { Heier et al. } \\
1997 \text { [30] }\end{array}$} & \multirow[t]{2}{*}{$\begin{array}{l}\text { Prospective } \\
\text { cohort study }\end{array}$} & \multirow{2}{*}{$\begin{array}{l}n=36 \\
\text { Overall mean age, } \\
16.3 \text { years } \\
\text { Overall age range, } \\
12.8-21.1 \text { years }\end{array}$} & $\begin{array}{l}-0.0175^{\prime \prime} \text { multistrand stainless steel } \\
\text { fixed retainer }(n=22)\end{array}$ & & \multirow[t]{2}{*}{6 months } & \multirow{2}{*}{$\begin{array}{l}\text { Maxillary and mandibular } \\
\text { anterior teeth } \\
\text { Maxillary and mandibular } \\
\text { dentition }\end{array}$} & \multirow{2}{*}{$\begin{array}{l}\text { Modified gingival index, } \\
\text { bleeding on probing, } \\
\text { Plaque Index, Calculus } \\
\text { Index, gingival crevicular } \\
\text { fluid flow }\end{array}$} \\
\hline & & & - Hawley retainer $(n=14)$ & Unclear & & & \\
\hline $\begin{array}{l}\text { Sobouti et al. } \\
2016 \text { [21] }\end{array}$ & $\mathrm{RCT}$ & $\begin{array}{l}n=128(60 \mathrm{M}, 68 \mathrm{~F}) \\
\text { Overall mean age, } \\
18 \pm 3.6 \text { years } \\
\text { Overall age range, } \\
13-25 \text { years }\end{array}$ & $\begin{array}{l}\text { - Fiber-reinforced composite fixed } \\
\text { retainer ( } n=42 \text {; mean age, } 18.5 \pm \\
3.6 \text { years) } \\
-0.0175^{\prime \prime} \text { flexible spiral wire fixed } \\
\text { retainer ( } n=41 \text {; mean age, } 18.4 \pm \\
3.7 \text { years) } \\
\text { - } 0.0009^{\prime \prime} \text { dead soft twisted wires } \\
\text { fixed retainer ( } n=45 \text {; mean age, } \\
17 \pm 3.3 \text { years) }\end{array}$ & & 24 months & $\begin{array}{l}\text { Mandibular anterior } \\
\text { teeth }\end{array}$ & Survival and failure rates \\
\hline $\begin{array}{l}\text { O'Rouke et al. } \\
2016 \text { [22] }\end{array}$ & $\mathrm{RCT}$ & $n=82(23 \mathrm{M}, 59 \mathrm{~F})$ & $\begin{array}{l}\text { - Vacuum-formed retainer ( } n=40 \text {, } \\
\text { mean age: } 16.95 \pm 2.02 \text { years) } \\
\text { - 0.0175" stainless steel coaxial } \\
\text { fixed retainer ( } n=42, \text { Mean age: } \\
18.47 \pm 4.41 \text { years) }\end{array}$ & $\begin{array}{l}\text { Full-time for } 6 \text { months, } \\
\text { followed by part-time for } \\
6 \text { months, then for every } \\
\text { other night in the second } \\
\text { year }\end{array}$ & 18 months & Mandibular dentition & $\begin{array}{l}\text { Little's irregularity index, } \\
\text { intercanine width, } \\
\text { intermolar width, arch } \\
\text { length, failure rate }\end{array}$ \\
\hline
\end{tabular}

${ }^{\mathrm{a} O v e r a l l ~ s a m p l e ~}$ 
of low risk of bias (Fig. 2) [12-14, 16-20, 22-24]. All six prospective cohort studies [25-30] (Fig. 3) were deemed to be of high quality in terms of sample selection, except for one study [25] which did not demonstrate the absence of pre-existing periodontal disease. Assessment of the outcome was deemed satisfactory in all but two studies [28, 29]. Overall, five prospective cohort studies were judged to be of moderate to high quality $[25-28,30]$.

\section{Periodontal outcomes}

Of the included trials, only seven trials assessed periodontal outcomes (Tables 2 and 3) [14, 16, 23, 25, 28, 30, 31]. Four of these were randomized controlled trials $[14,16,23,31]$, and the other three were prospective cohort studies
[25, 28, 30]. Two trials did not report baseline scores $[14,25]$, and another two studies reported the periodontal outcome with no distinction made between maxillary and mandibular measurements [30, 31].

No significant difference was found between mandibular stainless steel fixed retainers bonded to the anterior teeth and canines only in terms of periodontal outcomes, at 12-month and 3-year follow-ups in two studies $[25,28]$. With regard to periodontal outcomes of mandibular Hawley retainers in comparison to mandibular stainless steel fixed retainers, no significant difference was found at 3-year follow-up [28]. When mandibular fiber-reinforced composite was compared to mandibular stainless steel fixed retainers, no significant difference in probing depths, bleeding on probing, and calculus scores

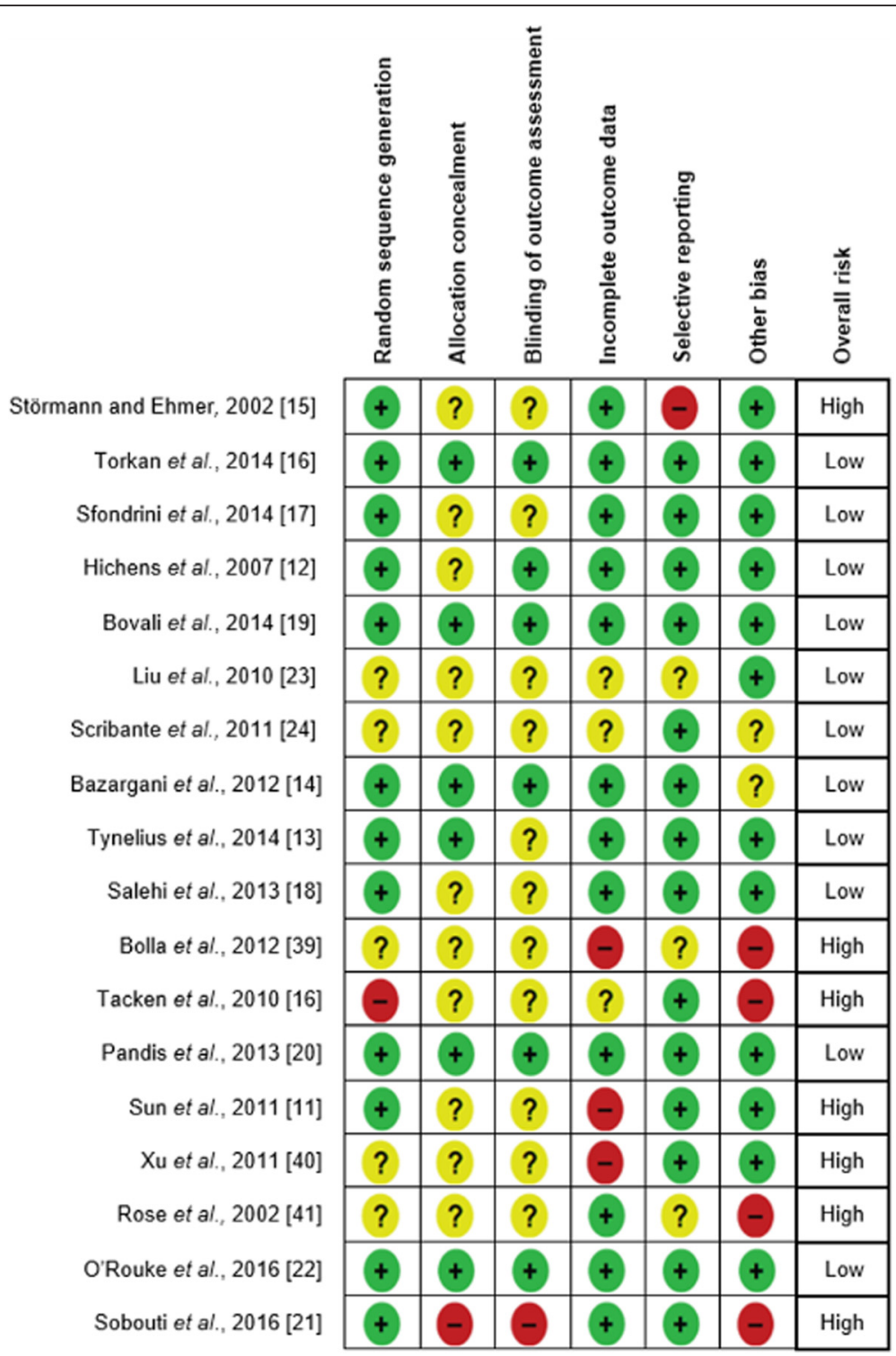

Fig. 2 Risk of bias for included randomized controlled trials. Low risk of bias (green). Unclear risk of bias (yellow). High risk of bias (red) 


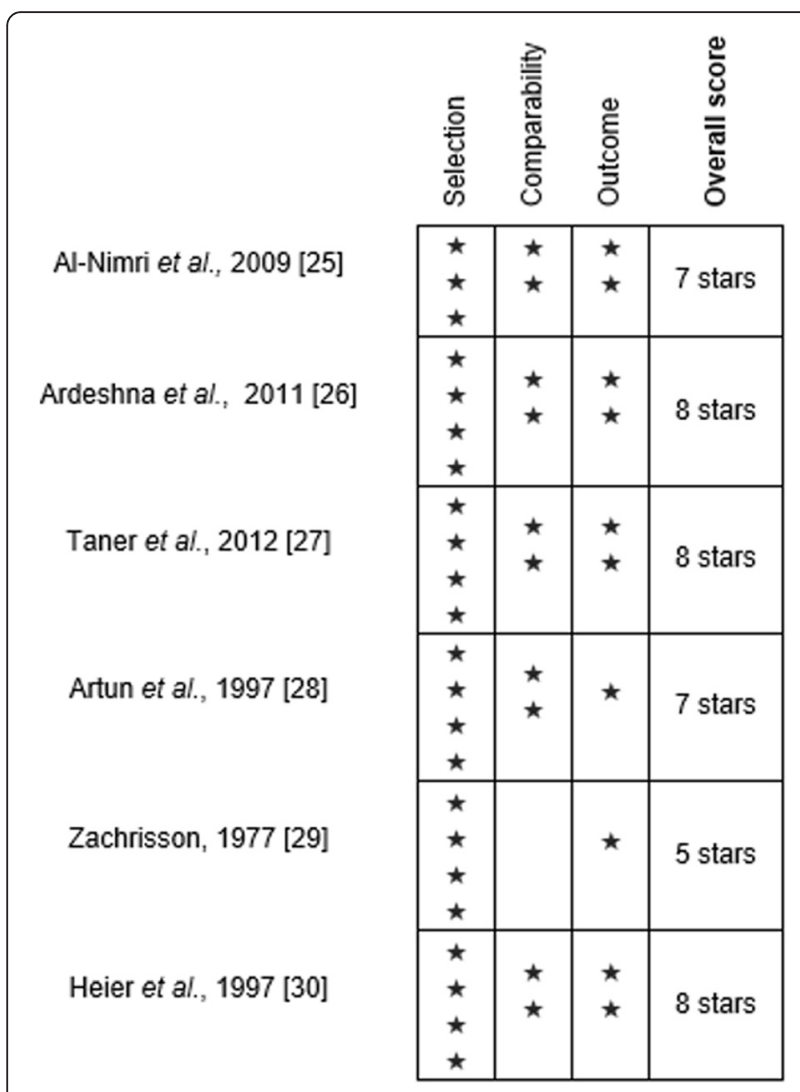

Fig. 3 Newcastle-Ottawa Scale scores for non-randomized studies

at 6-month follow-up was found [16, 23]. Probing depths and bleeding on probing were further measured at 12month follow-up and showed no significant difference between the two groups [23]. However, gingival and plaque indices scores were found to be higher in maxillary and mandibular fiber-reinforced composite compared to stainless steel fixed retainers at 6-month follow-up [16]. Very few overlapping studies were identified, however. Metaanalysis was therefore not possible in view of heterogeneity.

In terms of the natural history of periodontal changes related to stainless steel fixed retainers, plaque and gingival indices scores on the lingual surfaces of mandibular anterior teeth increased from baseline to 6 months followup; however, this was not statistically significant [16]. At 3-year follow-up, plaque and gingival indices scores remained low [28]. No significant changes in Calculus Index scores at 6-month [16] and 3-year follow-ups [28] were observed in two studies. Bleeding on probing scores for stainless steel fixed retainer increased at both 6 months $[16,23]$ and 12 months [23] from baseline, although only one study found this to be statistically significant [23]. Similar patterns were observed for fixed fiber-reinforced composite retainers $[16,23]$. Conversely, plaque, calculus, and gingival indices scores reduced at 3-year follow-up in relation to the lingual of the mandibular anterior teeth with Hawley retainers [28]. However, Gingival Index scores were shown to increase on the buccal surfaces of maxillary and mandibular anterior teeth in one study at 6-month follow-up [30].

\section{Survival and failure rates of retainers}

The survival rate of fixed retainers was reported over 12 to 24 months $[18,24,26]$. In terms of retainer material, one study found fiber-reinforced thermoplastic fixed retainer with polyethylene terephthalate glycol matrix resin survived significantly less than fiber-reinforced thermoplastic fixed retainer with polycarbonate matrix resin [26]. Two other studies found no significant difference in the survival rate of multistrand stainless steel fixed and esthetic retainers made of polyethylene woven ribbon or polyethelene fiber-reinforced resin composite $[18,24]$. No statistical difference was found in the survival rate between maxillary and mandibular fixed retainers $[18,26]$. Interestingly, in one study, the survival rate of fiber-reinforced thermoplastic fixed retainers was directly related to the thickness of the wire and the number of teeth bonded [26].

All the studies that involved mandibular stainless steel retainers reported failures per patient $[13,14,17-20$, $22-25,27,28]$, except for two studies in which the failure was reported per tooth [17, 24] (Table 4). The mean failure risk for mandibular stainless steel fixed retainers bonded to canine to canine was 0.29 (95\% confidence interval $[\mathrm{CI}], 0.26,0.33)$ based on nine studies $(n=555)$ (Fig. 4). The follow-up period ranged from 6 to 36 months. Similarly, the failure risk for mandibular stainless steel fixed retainers bonded to canines was 0.25 (95 \% CI, 0.16, 0.33) based on three studies [13, 25, 28] $(n=79)$ over a follow-up period of 12 to 36 months (Fig. 5). Considerable statistical heterogeneity was noted in both analyses ( $I$-squared $=89 \%)$ reflecting high levels of inconsistency and limited numbers of events. A metaregression shows that follow-up period was not a predictor of failure rate for mandibular stainless steel fixed retainers $(P=0.938)$.

One study reporting failure rates of mandibular Hawley retainers was unclear regarding the stipulated duration of wear [28]. However, two studies found around $12 \%$ failure over a period of 6 months and $14 \%$ at 3-year follow-up $[12,28]$. Similarly, the failure rate for maxillary vacuumformed retainers was found to be $10 \%$ over 2 years [13], while a further study reported a higher rate of $17 \%$ over 6 months [12].

\section{Patient-reported outcomes and cost-effectiveness}

Patient-reported outcomes were reported in two studies $[12,24]$ (Table 5). Removable retainers were found to be associated with discomfort, with those in the Hawley 
Table 2 Periodontal outcomes

\begin{tabular}{|c|c|c|c|c|c|c|}
\hline & Intervention & $\begin{array}{l}\text { Periodontal } \\
\text { outcomes }\end{array}$ & Index & Arch & Teeth & Tooth surfaces \\
\hline \multirow[t]{3}{*}{ Al-Nimri et al. 2009 [25] } & \multirow[t]{3}{*}{$\begin{array}{l}\text { - 0.036" round stainless steel fixed } \\
\text { retainer (canines only) } \\
-0.015^{\prime \prime} \text { multistrand fixed retainer }\end{array}$} & Plaque Index & $\begin{array}{l}0 \text { absence } \\
1 \text { on probe } \\
2 \text { visible } \\
3 \text { abundant }\end{array}$ & Mandible & $3-3$ & $\begin{array}{l}\text { Labial/lingual/mesial/ } \\
\text { distal }\end{array}$ \\
\hline & & Gingival Index & $\begin{array}{l}0 \text { absence } \\
1 \text { mild } \\
2 \text { moderate } \\
3 \text { severe }\end{array}$ & Mandible & $3-3$ & Labial and lingual \\
\hline & & Calculus & $\begin{array}{l}\text { Part of Oral Hygiene Index } \\
\text { Tooth with the highest score } \\
\text { determine the index score for } \\
\text { the segment ( } 6 \text { segments) }\end{array}$ & Maxilla and mandible & $\begin{array}{l}\text { All teeth except mandibular } \\
\text { labial segment }\end{array}$ & Labial and lingual \\
\hline Bazargani et al. 2012 [14] & $\begin{array}{l}-0.0195^{\prime \prime} \text { multistrand fixed retainer } \\
\text { with two-step bonded resin } \\
\text { adhesive } \\
-0.0195^{\prime \prime} \text { multistrand fixed retainer } \\
\text { with non-resin adhesive }\end{array}$ & Calculus & Present/absent & Mandible & $3-3$ & Lingual \\
\hline \multirow[t]{4}{*}{ Torkan et al. 2014 [16] } & \multirow[t]{4}{*}{$\begin{array}{l}\text { - Fiber-reinforced resin composite } \\
\text { fixed retainer } \\
\text { - } 0.0175 " \text { multistrand stainless } \\
\text { steel fixed retainer }\end{array}$} & Plaque Index & $\begin{array}{l}\text { Using disclosing } \\
0 \text { absence } \\
1 \text { visible on the probe } \\
2 \text { visible } \\
3 \text { abundant }\end{array}$ & Maxilla and mandible & $3-3$ & Lingual \\
\hline & & Calculus Index & $\begin{array}{l}0 \text { absence } \\
1 \text { up to } 1 / 3 \\
2 \text { up to } 2 / 3 \\
3>2 / 3\end{array}$ & Maxilla and mandible & All teeth & Unclear \\
\hline & & Gingival Index & $\begin{array}{l}0 \text { absence } \\
1 \text { mild } \\
2 \text { moderate } \\
3 \text { severe }\end{array}$ & Maxilla and mandible & Unclear & Lingual \\
\hline & & $\begin{array}{l}\text { Bleeding on } \\
\text { probing }\end{array}$ & Present/absent & Maxilla and mandible & $3-3$ & Unclear \\
\hline \multirow[t]{3}{*}{ Tacken et al. 2010 [31] } & \multirow[t]{3}{*}{$\begin{array}{l}\text { - Glass fiber-reinforced fixed retainer } \\
\text { (500 unidirectional glass fibers) } \\
\text { - Glass fiber-reinforced fixed retainer } \\
\text { (1000 unidirectional glass fibers) } \\
\text { - 0.0215" coaxial fixed retainer } \\
\text { - Untreated control }\end{array}$} & Gingival Index & $\begin{array}{l}0 \text { absence } \\
1 \text { mild (localized) } \\
2 \text { mild (generalized) } \\
3 \text { moderate } \\
4 \text { severe }\end{array}$ & Unclear & Unclear & $\begin{array}{l}\text { Unclear, } 3 \text { sites/tooth: } \\
\text { mesial, distal, central }\end{array}$ \\
\hline & & $\begin{array}{l}\text { Bleeding on } \\
\text { probing }\end{array}$ & $\begin{array}{l}0 \text { no bleeding } \\
1 \text { point bleeding } \\
2 \text { abundant bleeding }\end{array}$ & Unclear & Unclear & $\begin{array}{l}\text { Unclear, } 3 \text { sites/tooth: } \\
\text { mesial, distal, central }\end{array}$ \\
\hline & & Plaque Index & $\begin{array}{l}\text { Using disclosing } \\
0 \text { no plaque } \\
1 \text { spots at the cervical margin }\end{array}$ & Unclear & Unclear & $\begin{array}{l}\text { Unclear, } 3 \text { sites/tooth: } \\
\text { mesial, distal, central }\end{array}$ \\
\hline
\end{tabular}


Table 2 Periodontal outcomes (Continued)

\begin{tabular}{|c|c|c|c|c|c|c|}
\hline & & & $\begin{array}{l}2 \text { thin band at the cervical } \\
\text { margin } \\
3 \text { gingival } 1 / 3 \\
4 \text { gingival } 2 / 3 \\
5>\text { gingival } 2 / 3\end{array}$ & & & \\
\hline \multirow[t]{4}{*}{ Artun et al. 1997 [28] } & \multirow[t]{4}{*}{$\begin{array}{l}\text { - } 0.032^{\prime \prime} \text { plain fixed retainer (canines only) } \\
\text { - } 0.032^{\prime \prime} \text { spiral wire fixed retainer } \\
\text { (canines only) } \\
\text { - 0.0205" spiral wire fixed retainer } \\
\text { - Removable retainer }\end{array}$} & Plaque Index & $\begin{array}{l}0 \text { absence } \\
1 \text { on probe } \\
2 \text { visible } \\
3 \text { abundant }\end{array}$ & Mandible & $3-3$ & Lingual, mesial, distal \\
\hline & & Gingival Index & $\begin{array}{l}0 \text { absence } \\
1 \text { mild } \\
2 \text { moderate } \\
3 \text { severe }\end{array}$ & Mandible & $3-3$ & Lingual, mesial, distal \\
\hline & & Calculus Index & $\begin{array}{l}0 \text { absence } \\
1 \text { supragingival calculus not } \\
\text { more than } 1 \mathrm{~mm} \\
2 \text { gingival } 1 / 3 \\
3>\text { gingival } 2 / 3\end{array}$ & Mandible & $3-3$ & Lingual, mesial, distal \\
\hline & & Pocket depth & Mean attachment loss & Mandible & $3-3$ & Lingual \\
\hline \multirow[t]{2}{*}{ Liu et al. 2010 [23] } & \multirow{2}{*}{$\begin{array}{l}\text { - } 0.75 \text {-mm fiber-reinforced composite } \\
\text { fixed retainer } \\
\text { - 0.9-mm multistrand stainless steel } \\
\text { fixed retainer }\end{array}$} & Pocket depth & Scores added together & Mandible & $3-3$ & Lingual (3 sites/tooth) \\
\hline & & $\begin{array}{l}\text { Bleeding on } \\
\text { probing }\end{array}$ & Scores added together & Mandible & $3-3$ & Lingual (3 sites/tooth) \\
\hline \multirow[t]{4}{*}{ Heier et al. 2010 [30] } & \multirow[t]{4}{*}{$\begin{array}{l}\text { - } 0.0175^{\prime \prime} \text { multistrand stainless steel } \\
\text { fixed retainer } \\
\text { - Hawley retainer }\end{array}$} & Gingival Index & $\begin{array}{l}0 \text { absence } \\
1 \text { mild (localized) } \\
2 \text { mild (generalized) } \\
3 \text { moderate } \\
4 \text { severe }\end{array}$ & Maxilla and mandible & $3-3$ & $\begin{array}{l}\text { Labial, lingual, interdental } \\
\text { labial, interdental lingual }\end{array}$ \\
\hline & & $\begin{array}{l}\text { Bleeding on } \\
\text { probing }\end{array}$ & $\begin{array}{l}0 \text { absence } \\
1 \text { point bleeding } \\
2 \text { profuse }\end{array}$ & Maxilla and mandible & $3-3$ & $\begin{array}{l}\text { Labial, lingual, interdental } \\
\text { labial, interdental lingual }\end{array}$ \\
\hline & & Plaque Index & $\begin{array}{l}\text { Using disclosing } \\
0 \text { no plaque } \\
1 \text { spots at the cervical margin } \\
2 \text { thin band at the cervical } \\
\text { margin } \\
3 \text { gingival } 1 / 3 \\
4 \text { gingival } 2 / 3 \\
5>\text { gingival } 2 / 3\end{array}$ & Maxilla and mandible & $3-3$ & Labial, lingual \\
\hline & & Calculus Index & Overall mean score & Maxilla and mandible & $3-3$ & $\begin{array}{l}\text { Labial, lingual (3 sites/ } \\
\text { surface) }\end{array}$ \\
\hline
\end{tabular}


Table 3 Periodontal outcomes including the follow-up periods

\begin{tabular}{|c|c|c|c|c|c|c|c|c|c|}
\hline Study & Intervention & \multicolumn{4}{|l|}{ Plaque Index } & \multicolumn{4}{|l|}{ Gingival Index } \\
\hline \multirow[t]{2}{*}{ Al-Nimri et al. 2009 [25] } & $\begin{array}{l}\text { - 0.036" Round stainless } \\
\text { steel fixed retainer } \\
\text { (canines only) }(n=31)\end{array}$ & \multicolumn{4}{|c|}{$\begin{array}{l}\text { Mean after at least } 12 \text { months, } \\
1.02 \pm 0.52\end{array}$} & \multicolumn{4}{|c|}{$\begin{array}{l}\text { Mean after at least } 12 \text { months, } \\
1.19 \pm 0.44\end{array}$} \\
\hline & $\begin{array}{l}-0.015^{\prime \prime} \text { multistrand fixed } \\
\text { retainer }(n=31)\end{array}$ & \multicolumn{4}{|c|}{$\begin{array}{l}\text { Mean after at least } 12 \text { months, } \\
1.21 \pm 0.48\end{array}$} & \multicolumn{4}{|c|}{$\begin{array}{l}\text { Mean after at least } 12 \text { months, } \\
1.34 \pm 0.39\end{array}$} \\
\hline \multirow[t]{2}{*}{ Bazargani et al. 2012 [14] } & $\begin{array}{l}-0.0195 " \text { multistrand fixed } \\
\text { retainer with two-step } \\
\text { bonded resin adhesive } \\
(n=25)\end{array}$ & & & & & & & & \\
\hline & $\begin{array}{l}-0.0195 " \text { multistrand fixed } \\
\text { retainer with non-resin } \\
\text { adhesive }(n=26)\end{array}$ & & & & & & & & \\
\hline \multirow[t]{2}{*}{ Torkan et al. 2014 [16] } & $\begin{array}{l}\text { - Fiber-reinforced } \\
\text { composite resin fixed } \\
\text { retainer }(n=15)\end{array}$ & \multicolumn{4}{|c|}{$\begin{array}{l}\text { Maxilla: median } 0 \text { (baseline), } \\
1.66 \text { ( } 6 \text { months) } \\
\text { Mandible: median } 0.91 \text { (baseline), } \\
2 \text { ( } 6 \text { months) }\end{array}$} & \multicolumn{4}{|c|}{$\begin{array}{l}\text { Maxilla: median } 0.5 \text { (baseline), } \\
1 \text { ( } 6 \text { months) } \\
\text { Mandible: median } 0.33 \text { (baseline) } \\
1 \text { ( } 6 \text { months) }\end{array}$} \\
\hline & $\begin{array}{l}-0.0175^{\prime \prime} \text { multistrand } \\
\text { stainless steel fixed } \\
\text { retainer }(n=15)\end{array}$ & \multicolumn{4}{|c|}{$\begin{array}{l}\text { Maxilla: median } 0.33 \text { (baseline), } \\
0.66 \text { ( } 6 \text { months) } \\
\text { Mandible: median } 0.33 \text { (baseline), } \\
0.91 \text { ( } 6 \text { months) }\end{array}$} & \multicolumn{4}{|c|}{$\begin{array}{l}\text { Maxilla: median } 0 \text { (baseline), } \\
0.83 \text { ( } 6 \text { months) } \\
\text { Mandible: median } 0.16 \text { (baseline), } \\
0.41 \text { ( } 6 \text { months) }\end{array}$} \\
\hline \multirow[t]{3}{*}{ Tacken et al. 2010 [31] } & $\begin{array}{l}\text { - Glass fiber-reinforced } \\
\text { fixed retainer }(500 \\
\text { unidirectional glass } \\
\text { fibers) }(n=45)\end{array}$ & $\begin{array}{l}6 \text { months, } \\
1.88 \pm 0.74\end{array}$ & $\begin{array}{l}12 \text { months, } \\
2.32 \pm 0.93\end{array}$ & $\begin{array}{l}18 \text { months, } \\
2.25 \pm 0.78\end{array}$ & $\begin{array}{l}24 \text { months, } \\
2.11 \pm 0.73\end{array}$ & $\begin{array}{l}6 \text { months, } \\
1.20 \pm 0.43\end{array}$ & $\begin{array}{l}12 \text { months, } \\
1.00 \pm 0.30\end{array}$ & $\begin{array}{l}18 \text { months, } \\
1.28 \pm 0.36\end{array}$ & $\begin{array}{l}24 \text { months, } \\
1.51 \pm 0.45\end{array}$ \\
\hline & $\begin{array}{l}\text { - Glass fiber-reinforced } \\
\text { fixed retainer (1000 } \\
\text { unidirectional glass } \\
\text { fibers) }(n=48)\end{array}$ & $\begin{array}{l}6 \text { months, } \\
2.03 \pm 0.84\end{array}$ & $\begin{array}{l}12 \text { months, } \\
2.12 \pm 0.77\end{array}$ & $\begin{array}{l}18 \text { months, } \\
2.48 \pm 0.69\end{array}$ & $\begin{array}{l}24 \text { months, } \\
2.18 \pm 0.79\end{array}$ & $\begin{array}{l}6 \text { months, } \\
1.09 \pm 0.46\end{array}$ & $\begin{array}{l}12 \text { months, } \\
1.09 \pm 0.34\end{array}$ & $\begin{array}{l}18 \text { months, } \\
1.20 \pm 0.33\end{array}$ & $\begin{array}{l}24 \text { months, } \\
1.55 \pm 0.37\end{array}$ \\
\hline & $\begin{array}{l}-0.0215^{\prime \prime} \text { coaxial fixed } \\
\text { retainer }(n=91)\end{array}$ & $\begin{array}{l}6 \text { months, } \\
1.74 \pm 0.92\end{array}$ & $\begin{array}{l}12 \text { months, } \\
2.09 \pm 0.82\end{array}$ & $\begin{array}{l}18 \text { months, } \\
2.07 \pm 0.76\end{array}$ & $\begin{array}{l}24 \text { months, } \\
2.14 \pm 0.78\end{array}$ & $\begin{array}{l}6 \text { months, } \\
.0 .71 \pm 0.29\end{array}$ & $\begin{array}{l}12 \text { months, } \\
0.61 \pm 0.29\end{array}$ & $\begin{array}{l}18 \text { months, } \\
0.70 \pm 0.27\end{array}$ & $\begin{array}{l}24 \text { months, } \\
0.98 \pm 0.54\end{array}$ \\
\hline \multirow[t]{2}{*}{ Liu et al. 2010 [23] } & $\begin{array}{l}\text {-0.75-mm fiber-reinforced } \\
\text { composite fixed retainer } \\
(n=30)\end{array}$ & & & & & & & & \\
\hline & $\begin{array}{l}\text { - 0.9-mm multistrand } \\
\text { stainless steel fixed } \\
\text { retainer }(n=30)\end{array}$ & & & & & & & & \\
\hline \multirow[t]{2}{*}{ Artun et al. 1997 [28] } & $\begin{array}{l}-0.032^{\prime \prime} \text { plain fixed } \\
\text { retainer (canines only) } \\
(n=11)\end{array}$ & \multicolumn{4}{|c|}{$\begin{array}{l}\text { Baseline, } 0.32 \\
3 \text { years, } 0.06\end{array}$} & \multicolumn{4}{|l|}{$\begin{array}{l}\text { Baseline, } 1.01 \\
3 \text { years, } 0.66\end{array}$} \\
\hline & $\begin{array}{l}-0.032^{\prime \prime} \text { spiral fixed } \\
\text { retainer (canines only) } \\
(n=13)\end{array}$ & \multicolumn{4}{|c|}{$\begin{array}{l}\text { Baseline, } 0.17 \\
3 \text { years, } 0.10\end{array}$} & \multicolumn{4}{|l|}{$\begin{array}{l}\text { Baseline, } 0.95 \\
3 \text { years, } 0.49\end{array}$} \\
\hline
\end{tabular}


Table 3 Periodontal outcomes including the follow-up periods (Continued)

\begin{tabular}{llll}
\hline & $-0.0205 "$ spiral wire fixed & Baseline, 0.26 & Baseline, 1.14 \\
& retainer $(n=11)$ & 3 years, 0.13 & 3 years, 0.39 \\
& - Removable retainer & Baseline, 0.31 & Baseline, 1.08 \\
& $(n=14)$ & 3 years, 0.13 & 3 years, 0.77 \\
Heier et al. 2010 [30] & $-0.0175^{\prime \prime}$ multistrand & Baseline, 2.78 & Baseline, 0.79 \\
& stainless steel fixed & 6 months, 3.03 & 6 months, 0.40 \\
& retainer $(n=22)$ & & Baseline, 0.80 \\
& - Hawley retainer $(n=14)$ & Baseline, 2.78 & 6 months, 0.74 \\
\hline
\end{tabular}


Table 3 Periodontal outcomes including the follow-up periods (Continued)

\begin{tabular}{|c|c|c|c|c|c|c|}
\hline Study & Calculus & \multicolumn{4}{|c|}{ Bleeding on probing } & Probing attachment level \\
\hline \multicolumn{7}{|l|}{ Al-Nimri et al. 2009 [25] } \\
\hline \multirow[t]{2}{*}{ Bazargani et al. 2012 [14] } & $4 \%$ (2 years) & & & & & \\
\hline & $31 \%$ (2 years) & & & & & \\
\hline \multirow[t]{2}{*}{ Torkan et al. 2014 [16] } & $\begin{array}{l}\text { Maxilla: Median } 0 \text { (baseline } \\
\text { and } 6 \text { months) } \\
\text { Mandible: Median } 0 \text { (baseline), } \\
0.33 \text { (6 months) }\end{array}$ & \multicolumn{4}{|c|}{$\begin{array}{l}\text { Maxilla: Median } 0.16 \text { (baseline), } \\
0.5 \text { ( } 6 \text { months) } \\
\text { Mandible: Median } 0 \text { (baseline), } \\
0.66 \text { (6 months) }\end{array}$} & \\
\hline & $\begin{array}{l}\text { Maxilla and mandible: Median } 0 \\
\text { (baseline and } 6 \text { months) }\end{array}$ & \multicolumn{4}{|c|}{$\begin{array}{l}\text { Maxilla: median } 0 \text { (baseline), } \\
0.5 \text { ( } 6 \text { months) } \\
\text { Mandible: median } 0 \text { (baseline) } \\
0.33 \text { ( } 6 \text { months) }\end{array}$} & \\
\hline \multirow[t]{3}{*}{ Tacken et al. 2010 [31] } & & $\begin{array}{l}6 \text { months, } \\
0.72 \pm 0.22\end{array}$ & $\begin{array}{l}12 \text { months, } \\
0.89 \pm 0.19\end{array}$ & $\begin{array}{l}18 \text { month, } \\
0.82 \pm 0.23\end{array}$ & 24 months, $1.00 \pm 0.35$ & \\
\hline & & $\begin{array}{l}6 \text { months, } \\
0.76 \pm 0.18\end{array}$ & $\begin{array}{l}12 \text { months, } \\
0.81 \pm 0.21\end{array}$ & $\begin{array}{l}18 \text { months, } \\
0.89 \pm 0.23\end{array}$ & 24 months, $1.06 \pm 0.29$ & \\
\hline & & $\begin{array}{l}6 \text { months, } \\
0.46 \pm 0.18\end{array}$ & $\begin{array}{l}12 \text { months, } \\
0.55 \pm 0.19\end{array}$ & $\begin{array}{l}18 \text { months, } \\
0.57 \pm 0.21\end{array}$ & 24 months, $0.84 \pm 0.38$ & \\
\hline \multirow[t]{2}{*}{ Liu et al. 2010 [23] } & & $\begin{array}{l}\text { Baseline, } 3 \\
6 \text { months, } \\
12 \text { months }\end{array}$ & & & & $\begin{array}{l}\text { Baseline, } 6.33 \\
6 \text { months: } 8.51 \mathrm{~mm} \\
12 \text { months: } 9.24 \mathrm{~mm}\end{array}$ \\
\hline & & $\begin{array}{l}\text { Baseline, } 3 \\
8.89 ; 12 \mathrm{~m}\end{array}$ & $\begin{array}{l}\text { months, } \\
9.24\end{array}$ & & & $\begin{array}{l}\text { Baseline, } 5.92 \\
6 \text { months: } 8.08 \mathrm{~mm} \\
12 \text { months: } 8.92 \mathrm{~mm}\end{array}$ \\
\hline \multirow[t]{4}{*}{ Artun et al. 1997 [28] } & $\begin{array}{l}\text { Baseline, } 16.67 \\
3 \text { years, } 3.33\end{array}$ & & & & & Mean attachment loss at 3 years, $0.85 \mathrm{~mm}$ \\
\hline & $\begin{array}{l}\text { Baseline: } 8.64 \\
3 \text { years, } 3.09\end{array}$ & & & & & Mean attachment loss at 3 years, $0.63 \mathrm{~mm}$ \\
\hline & $\begin{array}{l}\text { Baseline, } 17.36 \\
3 \text { years, } 17.36\end{array}$ & & & & & Mean attachment loss at 3 years, $0.62 \mathrm{~mm}$ \\
\hline & $\begin{array}{l}\text { Baseline, } 9.52 \\
3 \text { years, } 8.33\end{array}$ & & & & & Mean attachment loss at 3 years, $0.72 \mathrm{~mm}$ \\
\hline \multirow[t]{2}{*}{ Heier et al. 2010 [30] } & Baseline and 6 months, 0.20 & \multicolumn{4}{|c|}{$\begin{array}{l}\text { Baseline, } 0.32 \\
6 \text { months, } 0.23\end{array}$} & \\
\hline & $\begin{array}{l}\text { Baseline, } 0.05 \\
6 \text { months, } 0.06\end{array}$ & \multicolumn{4}{|c|}{$\begin{array}{l}\text { Baseline, } 0.34 \\
6 \text { months, } 0.22\end{array}$} & \\
\hline
\end{tabular}


Table 4 Survival and failure rates of fixed and removable retainers

\begin{tabular}{|c|c|c|c|}
\hline Study & Intervention & Survival rate & Failure rate \\
\hline \multirow[t]{2}{*}{ Al-Nimri et al. 2009 [25] } & - 0.036" round stainless steel fixed retainer (canines only) & & $4 / 31(13 \%)$ \\
\hline & - 0.015" multistrand fixed retainer & & 9/31 (29\%) \\
\hline \multirow[t]{2}{*}{ Bazargani et al. 2012 [14] } & $\begin{array}{l}\text { - } 0.0195 " \text { multistrand fixed retainer with two-step } \\
\text { bonded resin adhesive }\end{array}$ & & $1 / 25(4 \%)$ \\
\hline & $\begin{array}{l}\text { - } 0.0195^{\prime \prime} \text { multistrand fixed retainer with non-resin } \\
\text { adhesive }\end{array}$ & & $7 / 26(27 \%)$ \\
\hline \multirow[t]{3}{*}{ Tynelius et al. 2014 [13] } & $\begin{array}{l}\text { - Vacuum-formed retainer in the maxilla and } 0.7-\mathrm{mm} \\
\text { spring hard wire fixed retainer in the mandible } \\
\text { (canines only) }\end{array}$ & & $\begin{array}{l}2 / 24(8.3 \%) \text { vacuum-formed retainer } \\
15 / 24(62.5 \%) \text { fixed retainer }\end{array}$ \\
\hline & $\begin{array}{l}\text { - Vacuum-formed retainer in the maxilla and } \\
\text { interproximal enamel reduction in the mandibular } \\
\text { anterior teeth }\end{array}$ & & $3 / 25(12 \%)$ \\
\hline & - Prefabricated positioner & & $0 / 25(0 \%)$ \\
\hline \multirow[t]{2}{*}{ Sfondrini et al. 2014 [17] } & $\begin{array}{l}\text { - } 0.5-\mathrm{mm} \text { silanized-treated glass fiber-reinforced } \\
\text { composite resin fixed retainer }\end{array}$ & & $27 / 240$ bonded teeth (11.25\%) \\
\hline & - 0.0175" multistrand stainless steel fixed retainer & & 50/282 bonded teeth (17.73\%) \\
\hline \multirow[t]{2}{*}{ Ardeshna et al. 2011 [26] } & $\begin{array}{l}\text { - } 0.53-\text { or } 1.02-\mathrm{mm} \text { fiber-reinforced thermoplastic fixed } \\
\text { retainer with polyethylene terephthalate glycol } \\
\text { matrix resin }\end{array}$ & Median, 2.97 months & 22/23 (95.6 \%) \\
\hline & $\begin{array}{l}\text { - } 0.53-\text { or } 1.02-\mathrm{mm} \text { fiber-reinforced thermoplastic fixed } \\
\text { retainer with polycarbonate matrix resin }\end{array}$ & Median, 11.37 months & $32 / 53(60.3 \%)$ \\
\hline \multirow[t]{2}{*}{ Salehi et al. 2013 [18] } & - Polyethylene woven ribbon fixed retainer & $\begin{array}{l}\text { Maxilla: mean } 13.96 \pm 4.53 \text { months } \\
\text { Mandible: mean } 14.26 \pm 4.70 \text { months }\end{array}$ & $\begin{array}{l}34 / 68 \text { in the maxilla }(50 \%), 29 / 68 \text { in } \\
\text { the mandible }(42.6 \%)\end{array}$ \\
\hline & - 0.0175" multistrand stainless steel fixed retainer & $\begin{array}{l}\text { Maxilla: mean } 15.34 \pm 4.04 \text { months } \\
\text { Mandible: mean } 15.61 \pm 3.61 \text { months }\end{array}$ & $\begin{array}{l}27 / 74 \text { in the maxilla }(36.5 \%), 28 / 74 \\
\text { in the mandible }(37.8 \%)\end{array}$ \\
\hline \multirow[t]{2}{*}{ Hichens et al. 2007 [12] } & - Hawley retainer & & 40/344 (11.6\%) \\
\hline & - Vacuum-formed retainer & & 20/366 (17\%) \\
\hline \multirow[t]{2}{*}{ Bovali et al. 2014 [19] } & $\begin{array}{l}\text { - Direct bonding of } 0.0215^{\prime \prime} \text { multistrand stainless steel } \\
\text { fixed retainer }\end{array}$ & & $7 / 29(24.1 \%)$ \\
\hline & $\begin{array}{l}\text { - Indirect bonding of } 0.0215^{\prime \prime} \text { multistrand stainless steel } \\
\text { fixed retainer }\end{array}$ & & 10/31 (32.2 \%) \\
\hline \multirow[t]{2}{*}{ Pandis et al. 2013 [20] } & $\begin{array}{l}\text { - } 0.022 " \text { multistrand stainless steel fixed retainer bonded } \\
\text { with chemical-cured composite }\end{array}$ & & $47 / 110(42.7 \%)$ \\
\hline & $\begin{array}{l}\text { - } 0.022^{\prime \prime} \text { multistrand stainless steel fixed retainer bonded } \\
\text { with light-cured composite }\end{array}$ & & $55 / 110(50 \%)$ \\
\hline \multirow[t]{2}{*}{ Liu et al. 2010 [23] } & - 0.75-mm fiber-reinforced composite fixed retainer & & $0 / 30(0 \%)$ \\
\hline & - 0.9-mm multistrand stainless steel fixed retainer & & $0 / 30(0 \%)$ \\
\hline Taner et al. 2012 [27] & $\begin{array}{l}\text { - Direct bonding } 0.016^{\prime \prime} \times 0.022^{\prime \prime} \text { multistrand stainless } \\
\text { steel dead soft wire fixed retainer }\end{array}$ & & 15/32 (46.8 \%) \\
\hline
\end{tabular}


Table 4 Survival and failure rates of fixed and removable retainers (Continued)

- Indirect bonding $0.016^{\prime \prime} \times 0.022^{\prime \prime}$ multistrand stainless

10/34 (29.4\%)

$1 / 11(9.1 \%)$

- 0.032" plain fixed retainer (canines only)

$4 / 13(30.7 \%)$

$3 / 11(27.27 \%)$

- $0.0205^{\prime \prime}$ spiral wire fixed retainer

$2 / 14(14.28 \%)$

Scribante et al. 2011 [24]

movable retainer

23/102 bonded teeth $(23 \%)$

multistrand stainless steel fixed retainer

$13 / 90$ bonded teeth $(14 \%)$ retainer

O'Rouke et al. 2016 [22]

- Vacuum-formed retainer

- 0.0175" stainless steel coaxial fixed retainer

$3 / 42(7.14 \%)$ 


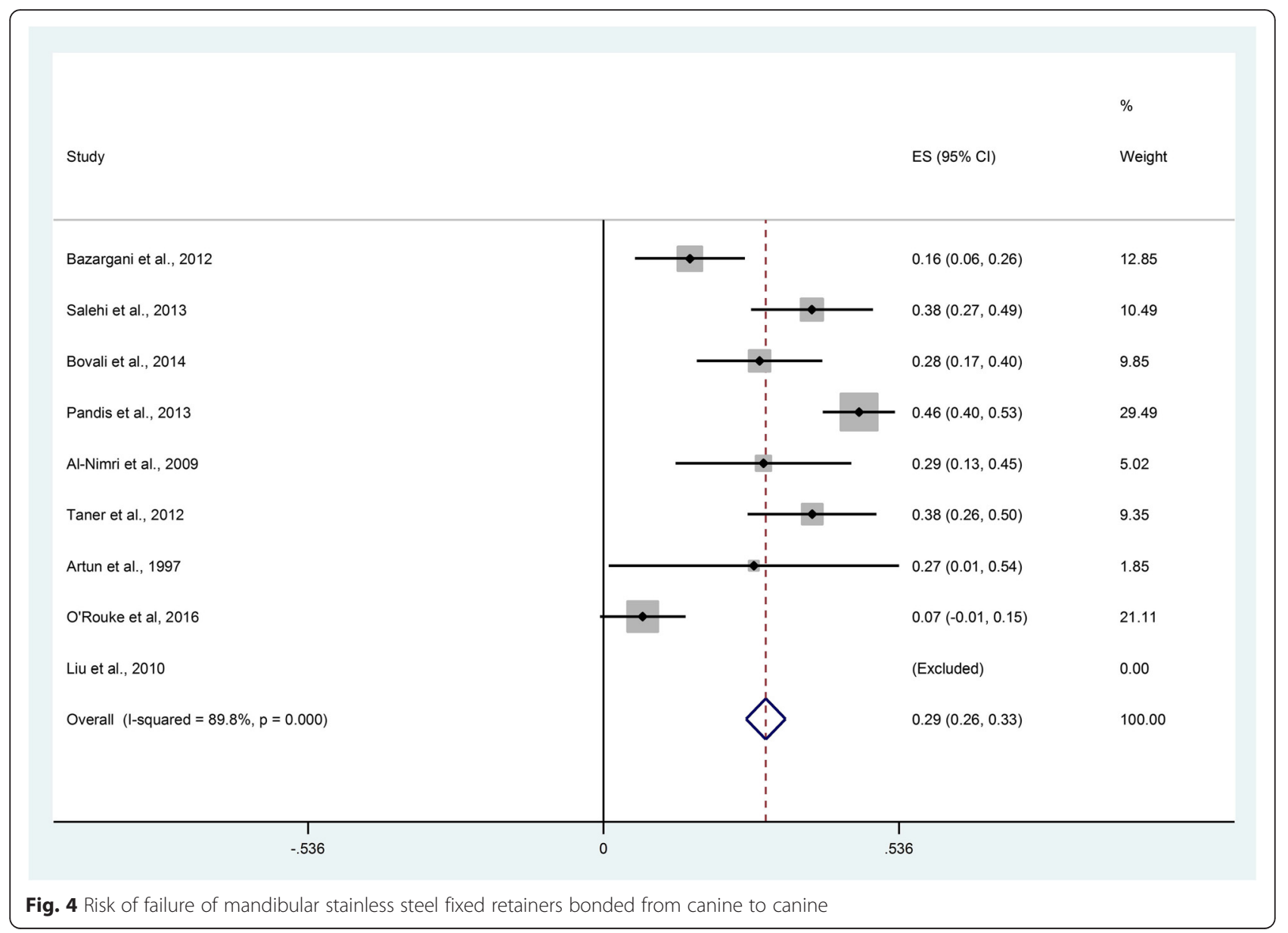

retainer group reporting higher levels of embarrassment in terms of speech and esthetics [12].

In terms of cost-effectiveness (Table 5), vacuumformed retainers were found to be significantly more cost-effective than Hawley retainers within the National Health Service over a 6-month retention period [12]. One study, over 2 years, found interproximal reduction as a retention method and positioners to be more costeffective than mandibular stainless steel fixed retainers bonded to canines [13].

\section{Discussion}

This systematic review found a lack of evidence to endorse the use of one type of orthodontic retainer based on their effect on periodontal health, survival and failure rates, patient-reported outcomes, and cost-effectiveness. Largely, this finding can be attributed to a lack of highquality, relevant research. In this respect, the results of the present systematic review are in line with previous systematic reviews $[3,9,10]$. Interestingly, it was found that failure of fixed stainless steel mandibular retainers was not directly related to the duration of follow-up. This suggests that other factors including the influence of operator technique and experience might override the effects of retainer design or materials, although followup did not extend beyond 3 years in the present review.

Generally, relatively minor changes in periodontal parameters were reported; however, given that most studies did not incorporate an untreated control, or indeed a control group without retention, it was unclear whether these changes were attributable to the intervention or temporal changes, in isolation. As such additional research including prospective cohort studies with matched controls incorporating baseline assessment would be helpful in providing more conclusive information. It is worthy of mention that the current standard of care is to recommend bonded retention to preserve orthodontic correction in those with a history of periodontal disease as these patients are known to be particularly susceptible to post-treatment changes [32, 33]. It is therefore important that there is greater clarity in relation to the compatibility of fixed retention with periodontal health and indeed on variations that may facilitate maintenance of optimal hygiene.

A minimum follow-up period of 6 months was set to distinguish between gingival inflammation associated 


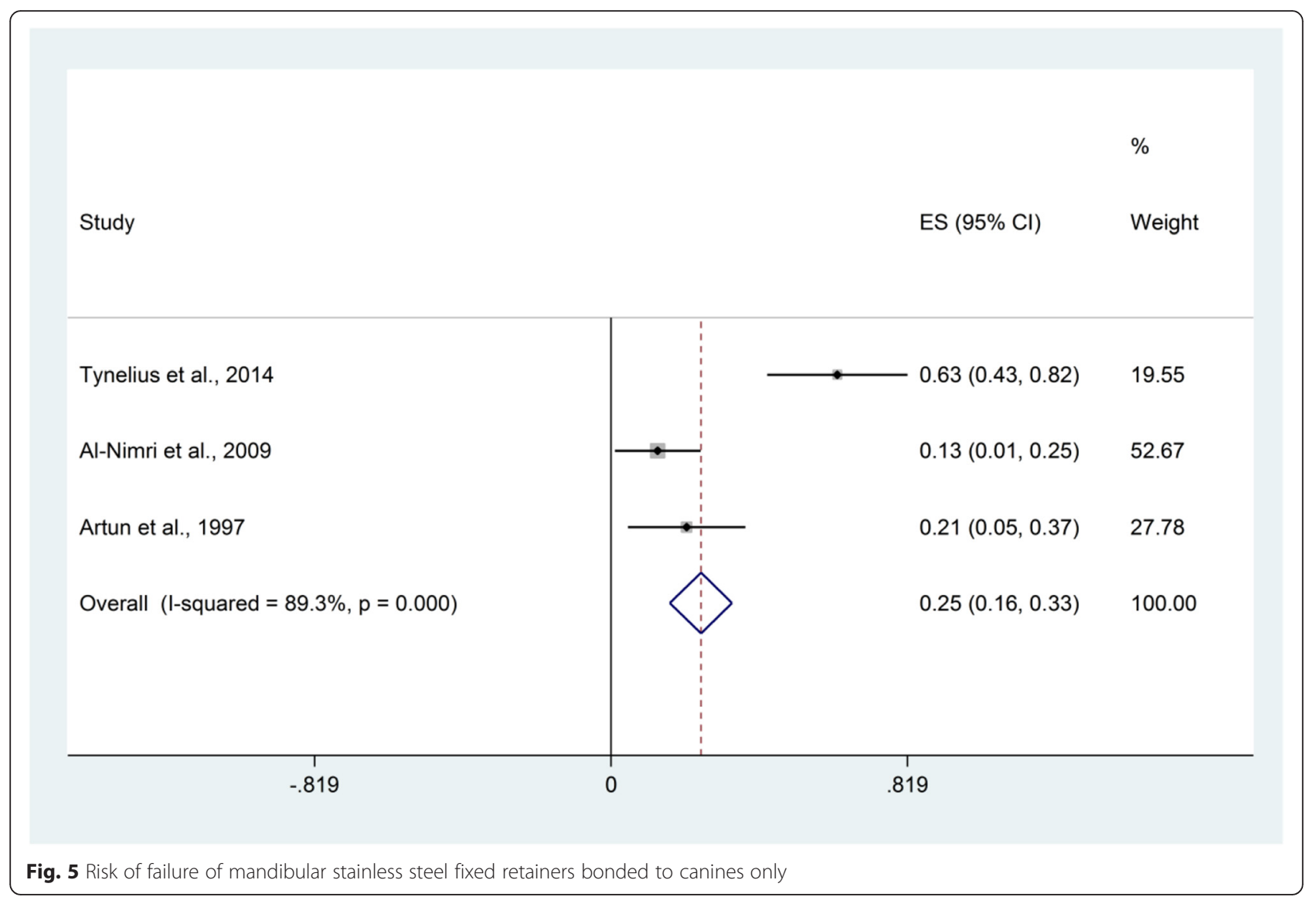

with fixed orthodontic treatment and periodontal sideeffects related to the orthodontic retainers [34]. Previous reviews have stipulated a minimum observation period of 3 months [3, 9] to 2 years [10]. However, a 3-month period might be insufficient to allow for the resolution of inflammatory changes related to the presence of active appliances. Using a minimum of 2-year observation period risks omission of a considerable amount of relevant research. Moreover, in this review, just one study focusing on periodontal outcomes involved follow-up in excess of 2 years [28]. It is therefore clear that the prolonged effect of orthodontic retention on periodontal health has not been adequately addressed in prospective research.

Intuitively, a significant difference in patient-reported outcomes and experiences could be expected with fixed or removable retainers in view of differences in appearance, size, and requirement for compliance. Notwithstanding this, only two studies reported on satisfaction with the appearance of retainers or on levels of associated embarrassment or discomfort [12, 24]. This tendency for researchers to concentrate on objective, often cliniciancentered outcomes has recently been documented both within orthodontics and general dental research more broadly $[35,36]$. Further studies incorporating patientreported outcomes are therefore necessary to provide a more holistic assessment of benefits, harms, and experiences associated with orthodontic retainers.

While the primary focus of this review was to compare the effectiveness of retainer types, it was also possible to generate epidemiological data on the risk of failure of fixed retainers based on the primary studies. Failure risk of 0.29 was found for fixed wires bonded to the six anterior teeth and approximately one-quarter of retainers bonded to mandibular canines only, based on observation periods of 6 months to 3 years. This data highlights that the risk of failure is considerable and that fixed retention does not guarantee prolonged stability. Similar findings have been observed in observational studies [2]. The onus on realistic treatment planning with due consideration for placement of teeth into a zone of relative stability therefore remains [37].

Attempts were made to identify all trials meeting the inclusion criteria in the present review with no restrictions based on either publication date or language. Furthermore, we planned to include both prospective cohort studies and randomized controlled trials. Cohort studies were included, in particular, to permit assessment of periodontal outcomes as they are more likely to involve more prolonged periods of follow-up, which may be necessary to reveal the extent of prolonged periodontal 
Table 5 Patient-reported outcomes and cost-effectiveness

\begin{tabular}{|c|c|c|c|c|}
\hline Study & Intervention & $\begin{array}{l}\text { Patient-reported } \\
\text { outcomes }\end{array}$ & Cost-effectiveness & \\
\hline \multirow[t]{3}{*}{ Tynelius et al. 2014 [13] } & $\begin{array}{l}\text { - Vacuum-formed retainer in the maxilla } \\
\text { and 0.7-mm spring hard wire fixed retainer } \\
\text { in the mandible (canines only) }\end{array}$ & & Costs of scheduled appointments, €12,425 & Costs of unscheduled appointments, $€ 804$ \\
\hline & $\begin{array}{l}\text { - Vacuum-formed retainer in the maxilla } \\
\text { and interproximal enamel reduction in } \\
\text { the mandibular anterior teeth }\end{array}$ & & Costs of scheduled appointments, €11,275 & Costs of unscheduled appointments, $€ 303$ \\
\hline & - Prefabricated positioner & & Costs of scheduled appointments, $€ 10,500$ & Costs of unscheduled appointments, none \\
\hline \multirow[t]{2}{*}{ Hichens et al. 2007 [12] } & - Hawley retainer & $\begin{array}{l}\text { Embarrassment: 29/168 (17 \%) } \\
\text { Discomfort: 109/168 (65 \%) }\end{array}$ & \multicolumn{2}{|c|}{$\begin{array}{l}\text { Mean cost to the NHS, } € 152(€ 150.86, € 153.15) \text { per patient } \\
\text { Mean cost to the orthodontic practice, }-€ 1.00(-€ 1.78,-€ 0.22) \text { per patient } \\
\text { Mean cost to the patient, } € 11.63(€ 9.67, € 13.59) \text { per patient }\end{array}$} \\
\hline & - Vacuum-formed retainer & $\begin{array}{l}\text { Embarrassment: 13/182 (7 \%) } \\
\text { Discomfort: 112/182 (62 \%) }\end{array}$ & \multicolumn{2}{|c|}{$\begin{array}{l}\text { Mean cost to the NHS, } € 122.02(€ 120.84, € 123.21) \text { per patient } \\
\text { Mean cost to the orthodontic practice, }-€ 34.00 \text { ( }-€ 34.57,-€ 33.34) \text { per patient } \\
\text { Mean cost to the patient, } € 6.92(€ 5.29, € 8.53) \text { per patient }\end{array}$} \\
\hline \multirow[t]{2}{*}{ Scribante et al. 2011 [24] } & $\begin{array}{l}\text { - } 0.0175 " \text { multistrand stainless steel } \\
\text { fixed retainer }\end{array}$ & $\begin{array}{l}\text { Mean, } 8.24 \pm 1.39 \text {; median, } 8.50 \text {; } \\
\text { range, }(4.50-10.0) \\
\text { (using visual analog scale) }\end{array}$ & & \\
\hline & $\begin{array}{l}\text { - Polyethylene fiber-reinforced resin } \\
\text { composite fixed retainer }\end{array}$ & $\begin{array}{l}\text { Mean, } 9.73 \pm 0.42 \text {; median, } 10.00 \text {; } \\
\text { range, }(9.00-10.0) \\
\text { (using visual analog scale) }\end{array}$ & & \\
\hline
\end{tabular}


effects. Meta-analysis was not undertaken in view of the clinical heterogeneity between the limited number of included studies, which made statistical pooling inappropriate in relation to periodontal health, survival and failure rates, patient-reported outcomes, and cost-effectiveness. This inability to undertake meta-analysis is common to many orthodontic systematic reviews with meta-analysis found in just $27 \%$ of 157 reviews over a 14-year period with a median of just 4 studies for those that did incorporate meta-analysis [38]. The onus on producing highquality primary research studies in orthodontics remains.

\section{Conclusions}

There is a lack of high-quality evidence to endorse the use of one type of orthodontic retainer based on their effect on periodontal health, risk of failure, patientreported outcomes, and cost-effectiveness. Further welldesigned prospective studies are therefore required to provide further definitive information in relation to the benefits and potential harms of prolonged retention.

\section{Appendix 1}

Database: Ovid MEDLINE(R) <1946 to Present>

Search Strategy:

1 RANDOMIZED CONTROLLED TRIAL.pt. (413632)

2 CONTROLLED CLINICAL TRIAL.pt. (91880)

3 RANDOM ALLOCATION.sh. (86446)

4 DOUBLE BLIND METHOD.sh. (135365)

5 SINGLE BLIND METHOD.sh. (21423)

6 or/1-5 (586980)

7 (ANIMALS not HUMANS).sh. (4033465)

8 CLINICAL TRIAL.pt. (506935)

9 exp Clinical Trial/ (849000)

10 (clin\$ adj25 trial\$).ti,ab. (308227)

11 ((singl\$ or doubl\$ or trebl\$ or tripl\$) adj25 (blind\$ or mask\$)).ti,ab. (146187)

12 PLACEBOS.sh. (34034)

13 placebo\$.ti,ab. (174121)

14 random\$.ti,ab. (804059)

15 RESEARCH DESIGN.sh. (84544)

16 or/9-15 (1594056)

1716 not $7(1478011)$

1817 not 8 (977433)

198 or $18(1484368)$

20 exp ORTHODONTICS/ (46224)

21 orthod\$.mp. (53863)

2220 or 21 (61325)

23 (retain\$ or retent\$).mp. (294935)

24 (fixed\$ or removable\$ or bonded\$ or Essix\$ or Hawley\$).mp. (221824)

2522 and 23 and 24 (1152)

2625 and 19 (174)

\section{Appendix 2}

Table 6 Excluded studies with reasons for exclusion $(n=36)$

\begin{tabular}{ll}
\hline Reason for exclusion & Studies \\
\hline Irrelative outcome to the systematic review & {$[42-63]$} \\
Cross sectional study & {$[64-67]$} \\
Follow-up less than 6 months & {$[68-70]$} \\
Subjects did not undergo orthodontic treatment & {$[71,72]$} \\
Retrospective study & {$[73-76]$} \\
No control group & {$[77]$} \\
\hline
\end{tabular}

Acknowledgements

Not applicable.

Funding

Not applicable.

Availability of data and materials

Data and materials supporting the findings are presented in the paper.

Authors' contributions

DA and PSF designed the systematic review and undertook the literature search and screening of the relevant studies, the data extraction, the quality assessment, the interpretation of the results, and the writing of the manuscript. NP undertook the statistical analysis and the interpretation. All three authors approved the submitted version.

\section{Competing interests}

The authors declare that they have no competing interests.

Consent for publication

Not applicable.

Ethics approval and consent to participate

Not applicable.

Author details

${ }^{1}$ Barts and The London School of Medicine and Dentistry, Queen Mary University of London, London E1 2AD, UK. 'Dental School, Medical Faculty, University of Bern, Bern, Switzerland.

Received: 28 May 2016 Accepted: 30 June 2016

Published online: 26 July 2016

References

1. Horowitz SL, Hixon EH. Physiologic recovery following orthodontic treatment. Am J Orthod. 1969;55(1):1-4.

2. Booth FA, Edelman JM, Proffit WR. Twenty-year follow-up of patients with permanently bonded mandibular canine-to-canine retainers. Am J Orthod Dentofac Orthop. 2008;133(1):70-6.

3. Littlewood SJ, Millett DT, Doubleday B, Bearn DR, Worthington HV. Retention procedures for stabilising tooth position after treatment with orthodontic braces. The Cochrane database of systematic reviews. 2016;1, CD002283.

4. Pratt MC, Kluemper GT, Hartsfield Jr JK, Fardo D, Nash DA. Evaluation of retention protocols among members of the American Association of Orthodontists in the United States. Am J Orthod Dentofac Orthop. 2011; 140(4):520-6.

5. Valiathan M, Hughes E. Results of a survey-based study to identify common retention practices in the United States. Am J Orthod Dentofac Orthop. 2010;137(2):170-7.

6. Wong PM, Freer TJ. A comprehensive survey of retention procedures in Australia and New Zealand. Aust Orthod J. 2004;20(2):99-106.

7. Renkema AM, Sips ET, Bronkhorst E, Kuijpers-Jagtman AM. A survey on orthodontic retention procedures in The Netherlands. Eur J Orthod. 2009;31(4):432-7. 
8. Case CS. Principles of retention in orthodontia. 1920. Am J Orthod Dentofac Orthop. 2003;124(4):352-61.

9. Littlewood SJ, Millett DT, Doubleday B, Bearn DR, Worthington HV. Orthodontic retention: a systematic review. J Orthod. 2006;33(3):205-12.

10. Westerlund A, Daxberg EL, Liljegren A, Oikonomou C, Ransjö M, Samuelsson O, Sjögren P. Stability and side effects of orthodontic retainers-a systematic review. Dentistry. 2014;2:2014.

11. Sun J, Yu YC, Liu MY, Chen L, Li HW, Zhang L, Zhou Y, Ao D, Tao R, Lai WL. Survival time comparison between Hawley and clear overlay retainers: a randomized trial. J Dent Res. 2011;90(10):1197-201.

12. Hichens $L$, Rowland $H$, Williams $A$, Hollinghurst S, Ewings $P$, Clark S, Ireland A, Sandy J. Cost-effectiveness and patient satisfaction: Hawley and vacuumformed retainers. Eur J Orthod. 2007;29(4):372-8.

13. Tynelius GE, Lilja-Karlander E, Petren S. A cost-minimization analysis of an RCT of three retention methods. Eur J Orthod. 2014;36(4):436-41.

14. Bazargani F, Jacobson S, Lennartsson B. A comparative evaluation of lingual retainer failure bonded with or without liquid resin. Angle Orthod. 2012;82(1):84-7

15. Störmann I, Ehmer U. A prospective randomized study of different retainer types. J Orofac Orthop. 2002;63(1):42-50.

16. Torkan S, Oshagh $M$, Khojastepour L, Shahidi S, Heidari S. Clinical and radiographic comparison of the effects of two types of fixed retainers on periodontium — a randomized clinical trial. Prog Orthod. 2014;15:47.

17. Sfondrini MF, Fraticelli D, Castellazzi L, Scribante A, Gandini P. Clinical evaluation of bond failures and survival between mandibular canine-tocanine retainers made of flexible spiral wire and fiber-reinforced composite. J Clin Exp Dent. 2014;6(2):e145-9.

18. Salehi P, Zarif Najafi H, Roeinpeikar SM. Comparison of survival time between two types of orthodontic fixed retainer: a prospective randomized clinical trial. Prog Orthod. 2013;14:25.

19. Bovali E, Kiliaridis S, Cornelis MA. Indirect vs direct bonding of mandibular fixed retainers in orthodontic patients: a single-center randomized controlled trial comparing placement time and failure over a 6-month period. Am J Orthod Dentofac Orthop. 2014;146(6): 701-8.

20. Pandis N, Fleming PS, Kloukos D, Polychronopoulou A, Katsaros C, Eliades T. Survival of bonded lingual retainers with chemical or photo polymerization over a 2-year period: a single-center, randomized controlled clinical trial. Am J Orthod Dentofac Orthop. 2013;144(2):169-75.

21. Sobouti F, Rakhshan V, Saravi MG, Zamanian A, Shariati M. Two-year survival analysis of twisted wire fixed retainer versus spiral wire and fiber-reinforced composite retainers: a preliminary explorative single-blind randomized clinical trial. Korean J Orthod. 2016;46(2):104-10.

22. O'Rourke N, Albeedh $\mathrm{H}$, Sharma P, Johal A. The effectiveness of bonded and vacuum formed retainers: a prospective randomized controlled clinical tria American journal of orthodontics and dentofacial orthopedics, "In Press".

23. Liu Y. Application of fiber-reinforced composite as fixed lingual retainer. Hua Xi Kou Qiang Yi Xue Za Zhi. 2010;28(3):290-3.

24. Scribante A, Sfondrini MF, Broggini S, D'Allocco M, Gandini P. Efficacy of esthetic retainers: clinical comparison between multistranded wires and direct-bond glass fiber-reinforced composite splints. Int J Dent. 2011;2011:548356.

25. Al-Nimri K, Al Habashneh R, Obeidat M. Gingival health and relapse tendency: a prospective study of two types of lower fixed retainers. Aust Orthod J. 2009;25(2):142-6.

26. Ardeshna AP. Clinical evaluation of fiber-reinforced-plastic bonded orthodontic retainers. Am J Orthod Dentofac Orthop. 2011;139(6):761-7.

27. Taner T, Aksu M. A prospective clinical evaluation of mandibular lingual retainer survival. Eur J Orthod. 2012;34(4):470-4.

28. Artun J, Spadafora AT, Shapiro PA. A 3-year follow-up study of various types of orthodontic canine-to-canine retainers. Eur J Orthod. 1997:19(5):501-9.

29. Zachrisson BU. Clinical experience with direct-bonded orthodontic retainers. Am J Orthod. 1977:71(4):440-8.

30. Heier EE, De Smit AA, Wijgaerts IA, Adriaens PA. Periodontal implications of bonded versus removable retainers. Am J Orthod Dentofac Orthop. 1997; 112(6):607-16

31. Tacken MP, Cosyn J, De Wilde P, Aerts J, Govaerts E, Vannet BV. Glass fibre reinforced versus multistranded bonded orthodontic retainers: a 2 year prospective multi-centre study. Eur J Orthod. 2010;32(2):117-23.

32. Clinical Guidelines: Orthodontic Retention. Published 2008, revised 2013. Available at: https://www.rcseng.ac.uk/fds/publications-clinical-guidelines/ clinical_guidelines. Accessed May 092016.
33. Sharpe W, Reed B, Subtelny JD, Polson A. Orthodontic relapse, apical root resorption, and crestal alveolar bone levels. American journal of orthodontics and dentofacial. 1987:91(3):252-8.

34. Zachrisson S, Zachrisson BU. Gingival condition associated with orthodontic treatment. Angle Orthod. 1972;42(1):26-34.

35. Tsichlaki A, O'Brien K. Do orthodontic research outcomes reflect patient values? A systematic review of randomized controlled trials involving children. Am J Orthod Dentofac Orthop. 2014;146(3):279-85.

36. Fleming PS, Koletsi D, O'Brien K, Tsichlaki A, Pandis N. Are dental researchers asking patient-important questions? A scoping review. J Dent. 2016;49:9-13.

37. Lee RT. Arch width and form: a review. Am J Orthod Dentofac Orthop. 1999;115(3):305-13.

38. Koletsi D, Fleming PS, Eliades T, Pandis N. The evidence from systematic reviews and meta-analyses published in orthodontic literature. Where do we stand? Eur J Orthod. 2015:37(6):603-9.

39. Bolla E, Cozzani M, Doldo T, Fontana M. Failure evaluation after a 6-year retention period: a comparison between glass fiber-reinforced (GFR) and multistranded bonded retainers. Int Orthod. 2012;10(1):16-28

40. Xu XC, Li RM, Tang GH. Clinical evaluation of lingual fixed retainer combined with Hawley retainer and vacuum-formed retainer. Shanghai Kou Qiang Yi Xue. 2011;20(6):623-6.

41. Rose E, Frucht S, Jonas IE. Clinical comparison of a multistranded wire and a direct-bonded polyethylene ribbon-reinforced resin composite used for lingual retention. Quintessence Int. 2002;33(8):579-83.

42. Tecco S, Epifania E, Festa F. An electromyographic evaluation of bilateral symmetry of masticatory, neck and trunk muscles activity in patients wearing a positioner. J Oral Rehabil. 2008;35(6):433-9.

43. Greco PM, Vanarsdall Jr RL, Levrini M, Read R. An evaluation of anterior temporal and masseter muscle activity in appliance therapy. Angle Orthod. 1999:69(2):141-6.

44. Schott TC, Goz G. Applicative characteristics of new microelectronic sensors Smart Retainer(R) and TheraMon(R) for measuring wear time. J Orofac Orthop. 2010;71(5):339-47.

45. Aslan BI, Dincer M, Salmanli O, Qasem MA. Comparison of the effects of modified and full-coverage thermoplastic retainers on occlusal contacts. Orthodontics (Chic). 2013;14(1):e198-208.

46. Edman Tynelius G, Bondemark L, Lilja-Karlander E. A randomized controlled trial of three orthodontic retention methods in class I four premolar extraction cases - stability after 2 years in retention. Orthod Craniofac Res. 2013;16(2):105-15.

47. Tynelius GE, Bondemark L, Lilja-Karlander E. Evaluation of orthodontic treatment after 1 year of retention - a randomized controlled trial. Eur J Orthod. 2010;32(5):542-7.

48. Tynelius GE, Petren S, Bondemark L, Lilja-Karlander E. Five-year postretention outcomes of three retention methods-a randomized controlled trial. Eur J Orthod. 2015;37(4):345-53.

49. Shawesh M, Bhatti B, Usmani T, Mandall N. Hawley retainers full- or part-time? A randomized clinical trial. Eur J Orthod. 2010;32(2):165-70.

50. Sari Z, Uysal T, Basciftci FA, Inan O. Occlusal contact changes with removable and bonded retainers in a 1-year retention period. Angle Orthod. 2009:79(5):867-72

51. Gill DS, Naini FB, Jones A, Tredwin CJ. Part-time versus full-time retainer wear following fixed appliance therapy: a randomized prospective controlled trial. World J Orthod. 2007;8(3):300-6.

52. Bauer EM, Behrents R, Oliver DR, Buschang PH. Posterior occlusion changes with a Hawley vs Perfector and Hawley retainer. A follow-up study. Angle Orthod. 2010;80(5):853-60.

53. Ackerman MB, Thornton B. Posttreatment compliance with removable maxillary retention in a teenage population: a short-term randomized clinical trial. Orthodontics (Chic). 2011;12(1):22-7.

54. Wiedel AP, Bondemark L. Stability of anterior crossbite correction: a randomized controlled trial with a 2-year follow-up. Angle Orthod. 2015;85(2):189-95.

55. Rowland $H$, Hichens L, Williams A, Hills D, Killingback N, Ewings P, Clark S, Ireland AJ, Sandy JR. The effectiveness of Hawley and vacuum-formed retainers: a single-center randomized controlled trial. Am J Orthod Dentofac Orthop. 2007;132(6):730-7.

56. Thickett E, Power S. A randomized clinical trial of thermoplastic retainer wear. Eur J Orthod. 2010:32(1):1-5.

57. Sauget E, Covell Jr DA, Boero RP, Lieber WS. Comparison of occlusal contacts with use of Hawley and clear overlay retainers. Angle Orthod. 1997;67(3):223-30. 
58. Dincer $M$, Isik AB. Effects of thermoplastic retainers on occlusal contacts. Eur J Orthod. 2010;32(1):6-10.

59. Turkoz C, Canigur Bavbek N, Kale Varlik S, Akca G. Influence of thermoplastic retainers on Streptococcus mutans and Lactobacillus adhesion. Am J Orthod Dentofac Orthop. 2012;141(5):598-603.

60. Basciftci FA, Uysal T, Sari Z, Inan O. Occlusal contacts with different retention procedures in 1-year follow-up period. Am J Orthod Dentofac Orthop. 2007; 131(3):357-62.

61. Hyun P, Preston CB, Al-Jewair TS, Park-Hyun E, Tabbaa S. Patient compliance with Hawley retainers fitted with the SMART((R)) sensor: a prospective clinical pilot study. Angle Orthod. 2015;85(2):263-9.

62. Andren A, Naraghi S, Mohlin BO, Kjellberg H. Pattern and amount of change after orthodontic correction of upper front teeth 7 years postretention. Angle Orthod. 2010;80(4):432-7.

63. Schott TC, Schlipf C, Glasl B, Schwarzer CL, Weber J, Ludwig B. Quantification of patient compliance with Hawley retainers and removable functional appliances during the retention phase. Am J Orthod Dentofac Orthop. 2013; 144(4):533-40

64. Pandis N, Vlahopoulos K, Madianos P, Eliades T. Long-term periodontal status of patients with mandibular lingual fixed retention. Eur J Orthod. 2007;29(5):471-6.

65. Moffitt AH, Raina J. Long-term bonded retention after closure of maxillary midline diastema. Am J Orthod Dentofac Orthop. 2015;148(2):238-44.

66. Dietrich P, Patcas R, Pandis N, Eliades T. Long-term follow-up of maxillary fixed retention: survival rate and periodontal health. Eur J Orthod. 2015; 37(1):37-42.

67. César Neto JB, Régio MRS, Martos J, Spautz F, Moraes GB. Analysis of the periodontal status of patients with mandibular-bonded retainers. Revista Odonto Ciência. 2010;25:132-6.

68. Horton JK, Buschang PH, Oliver DR, Behrents RG. Comparison of the effects of Hawley and perfector/spring aligner retainers on postorthodontic occlusion. Am J Orthod Dentofac Orthop. 2009;135(6):729-36.

69. Kumar AG, Bansal A. Effectiveness and acceptability of Essix and Begg retainers: a prospective study. Aust Orthod J. 2011;27(1):52-6.

70. Artun J, Spadafora AT, Shapiro PA, McNeill RW, Chapko MK. Hygiene status associated with different types of bonded, orthodontic canine-to-canine retainers. A clinical trial. J Clin Periodontol. 1987;14(2):89-94.

71. Hohoff A, Stamm T, Goder G, Sauerland C, Ehmer U, Seifert E. Comparison of 3 bonded lingual appliances by auditive analysis and subjective assessment. Am J Orthod Dentofac Orthop. 2003;124(6):737-45.

72. Meade MJ, Millett DT, Cronin M. Social perceptions of orthodontic retainer wear. Eur J Orthod. 2014;36(6):649-56.

73. Tsomos G, Ludwig B, Grossen J, Pazera P, Gkantidis N. Objective assessment of patient compliance with removable orthodontic appliances: a crosssectional cohort study. Angle Orthod. 2014;84(1):56-61.

74. Kuijpers MA, Kiliaridis S, Renkema A, Bronkhorst EM, Kuijpers-Jagtman AM. Anterior tooth wear and retention type until 5 years after orthodontic treatment. Acta Odontol Scand. 2009;67(3):176-81.

75. Hoybjerg AJ, Currier GF, Kadioglu O. Evaluation of 3 retention protocols using the American Board of Orthodontics cast and radiograph evaluation. Am J Orthod Dentofac Orthop. 2013;144(1):16-22.

76. Tang AT, Forsberg CM, Andlin-Sobocki A, Ekstrand J, Hagg U. Lingual retainers bonded without liquid resin: a 5-year follow-up study. Am J Orthod Dentofac Orthop. 2013;143(1):101-4.

77. Jaderberg S, Feldmann I, Engstrom C. Removable thermoplastic appliances as orthodontic retainers-a prospective study of different wear regimens. Eur J Orthod. 2012;34(4):475-9.

\section{Submit your manuscript to a SpringerOpen ${ }^{\odot}$ journal and benefit from:}

- Convenient online submission

- Rigorous peer review

- Immediate publication on acceptance

- Open access: articles freely available online

- High visibility within the field

- Retaining the copyright to your article 